\title{
Towards simulation of force and velocity fluctuations due to turbulence in the relay nozzle jet of an air jet loom
}

\begin{abstract}
This research was aimed at obtaining a first estimation of the effect of turbulent vortices present in the relay nozzle jets of an air jet loom on the weft. To this end a large eddy simulation (LES) model was set up and validated capable of simulating a highly underexpanded jet up to a point sufficiently far from the nozzle exit such that flow features at the weft location could be analyzed. The quality of the LES was evaluated based on several quality criteria as well as by comparing the results to experiments and data from literature. The results show that for a free jet substantial velocity fluctuations are present at a representative yarn location. By inserting a rigid cylinder at this location, the corresponding force fluctuations on a smooth yarn were also obtained. The research shows that the unsteadiness in the jet is quite substantial, as are the corresponding force fluctuations. These fluctuations could have a profound impact on the yarn motion and should at least be considered when using numerical tools to evaluate the forces on or the motion of a yarn acted on by a relay nozzle jet.
\end{abstract}

\section{Keywords}

Air jet loom, Relay nozzle, Computational fluid dynamics (CFD), Large eddy simulation (LES)

\section{Introduction}

In air jet looms, a series of air jets is used to propel a yarn (the weft) across the width of a fabric. By using jets, no mechanical components have to travel back and forth along the width of the machine and a high production speed can be attained. Because of their high productivity, these machines are often used for mass production of fabrics. ${ }^{1}$ Unfortunately, the absence of a mechanical component acting directly on the yarn makes the yarn motion and the forces it experiences harder to predict. When the yarn breaks due to excessive tension or when it is blown out of the reed channel, a failed insertion occurs. This requires a restart of the machine and lowers the productivity. ${ }^{1,2}$

The weft first passes through a main nozzle or a tandem of main nozzles. These nozzles are supplied with highly pressurized air, generating a high-speed air flow which pulls the weft from the storage device and blows the weft into the reed channel. The reed consists of a series of profiled lamellae forming a channel for the weft to pass through. Relay nozzles are positioned at regular intervals along the entire length of this channel. These relay nozzles generate high-speed air jets, which are directed towards the reed channel at an acute angle with respect to it (and consequently the yarn). The air flow from the main nozzles and the relay nozzles is channeled by the reed, in order to obtain a better transfer of energy from the air flow to the yarn as well as a better guidance of the yarn. After some distance the air flow in the reed channel is determined solely by the relay nozzle jets and not influenced anymore by the flow from the main nozzles. A more detailed discussion of the insertion process and the components of an air jet loom (and their functionality) can be found in the work of Vangheluwe. ${ }^{3}$ 
Over the past years, the modelling of the yarn motion and/or tension has attracted quite some interest with the goal of increasing the efficiency and productivity of the machine. Initially, these models were based on analytical and empirical formulas. Uno ${ }^{4}$ was among the first to model the yarn dynamics in an air jet loom. More specifically, he combined an analytical model for the yarn unwinding force with an empirical model for the main nozzle flow. With his model he estimated the velocity of a yarn as it is launched by a main nozzle. In that research it was assumed that the yarn remained straight and moved along the axis of the main nozzle. The force exerted by the air flow on the yarn was calculated based on empirically determined force coefficients. A similar method was, for example, applied by Nosraty, Jedi and Mousaloo ${ }^{5}$ who focused on calculation of the yarn velocity and/or tension. One of the first investigations to consider the effect of relay nozzle jets was that of Celik, Babaarslan and Bandara. ${ }^{6}$

Compared to the original designs, the productivity of air jet looms has increased considerably but so has the complexity of the air flow. Consequently, numerical investigations have become more prominent in the study of air jet looms. Numerical investigations of the air flow are usually performed with computational fluid dynamics (CFD). Among the first to apply CFD to air jet looms were Oh, Kim and Song ${ }^{7}$. They performed simulations of the air flow inside several main nozzle geometries in order to obtain some basic design guidelines. Other examples of CFD investigations of the main nozzle are the works of Kim, Lim, Lee et al. ${ }^{8}$, Belforte, Mattiazio, Viktorov et al. ${ }^{9}$, Jin, Cui, Zhu et al. ${ }^{10}$ and He, Qian, Xue et al. ${ }^{11}$. Not only the main nozzle but also the relay nozzles have been the subject of CFD investigations, albeit to a lesser extent. By means of CFD, Kim, Setoguchi and $\mathrm{Kim}^{12}$ studied the flow field in the reed channel for several relay nozzle diameters, supply pressures and orientations. Grassi, Schröter, Gloy et al. ${ }^{13}$ and Münkel, Gloy, Gries et al. ${ }^{14}$ performed CFD simulations to test and evaluate a new energy-efficient design for the relay nozzles.

The research mentioned above has mainly focused on the axial yarn motion and the axial force exerted on the yarn. However, for a weft insertion the radial motion of the yarn can be equally important. On the one hand, the radial motion will influence the tension in the yarn and the friction of the yarn with the reed. On the other hand, to avoid a failed insertion, the weft has to remain within the reed channel. Keeping the yarn within the reed channel is one of the main functionalities of the relay nozzles. Currently, the position and orientation of relay nozzles is tuned based on experience to obtain a reliable insertion. However, occasionally the yarn still leaves the reed channel. To the best of the authors' knowledge, no models currently exist that can provide information about the radial oscillations of the yarn during weft insertion.

In an air jet loom, the dynamics of the yarn are largely determined by the interaction between the air flow and the yarn. To analyze the interaction between a complex flow (such as the air flow in an air jet loom) and a flexible structure, one typically has to resort to fluid-structure interaction (FSI) simulations. Unfortunately, FSI simulations are quite resource-intensive and therefore still quite scarce. For air jet looms the high air velocity and high aspect ratio of the yarn pose additional complications for these simulations. Nevertheless, FSI-simulations have already been used to study air-yarn interaction ${ }^{2,15-20}$. Concerning air jet looms the FSI research has mainly focused on the interaction between the main nozzle flow and the weft. To the best of the authors' knowledge no FSI research has been performed on the interaction between a weft and the flow from the relay nozzles. However, as mentioned above, the interaction between the relay nozzle flow and the weft is essential to keeping the yarn within the reed channel as well as possible.

FSI simulations involving the weft and the main nozzle have mainly relied on Reynolds-averaged NavierStokes (RANS) simulations for calculation of the flow field. In RANS simulations the turbulent fluctuations of the flow are averaged out and are consequently not resolved. Similarly, in the previously mentioned CFD research concerning the relay nozzles, RANS models were employed and turbulent fluctuations in the jet were not considered. However, contrary to the interaction between the weft and the main nozzle flow the 
interaction with the relay nozzle flow occurs in a less confined space, at some distance from the nozzle exit and further removed from the centerline of the jet, giving turbulent vortices time and space to grow. These turbulent vortices could result in substantial unsteady forces exerted on the weft and thereby an erratic motion of the weft, especially near the tip, where the tension in the yarn is limited. One possibility to obtain information about these turbulent oscillations and their influence is to resort to large eddy simulations (LES). ${ }^{21}$ This methodology is based on spatial filtering of the Navier-Stokes equations and only models small turbulent structures while resolving the larger ones. Unfortunately, the computational cost of an LES is considerably higher than that of a RANS simulation. Furthermore, the modelling of high-pressure, highvelocity jets presents additional challenges related to the compressibility of the fluid. One work that considers the interaction between a fiber launched by the main nozzle and the turbulent fluctuations in the main nozzle jet is that of Lin et al. ${ }^{22}$. In that work the flow field behind the main nozzle exit was simulated by means of a 2D, planar LES. From the flow field, which thus included turbulent fluctuations, the forces experienced by the yarn were calculated using a drag coefficient, formulated as a function of the Reynolds number. Using these forces the motion of the fiber was calculated. Based on their simulations they put forward that for fibers with a higher linear density the first relay nozzle could be placed further from the main nozzle.

The main goal of this paper is to set up and validate an LES framework to simulate highly underexpanded jets (as often encountered in relay nozzle flow) up to a point sufficiently far from the nozzle exit such that it covers the region where a relay nozzle jet would interact with the weft in order to create awareness of the turbulent fluctuations present in the relay nozzle jet and to provide an idea about the required computational resources to perform such simulations. Additionally, an initial assessment of the magnitude of the turbulent velocity fluctuations at this distance from the nozzle exit and the resulting forces on a weft-like structure are provided.

The importance for the textile research field is that these chaotic fluctuations can influence the behavior of the weft during insertion. The flow oscillations can affect the tension in the yarn and thereby the yarn breakage. These fluctuations can also influence the lateral motion of the yarn, affecting the number of weaving faults and missed insertions (when the yarn is blown out of the reed channel). The current work is considered as a step towards the numerical modelling of these turbulent fluctuations and their effect on the weft. Eventually, such numerical simulations can yield a better understanding of the weft behavior during insertion. Analysis of the data from such simulations supplemented with experiments can lead to new and/or improved criteria for yarn breakage and missed insertions, improved designs for the relay nozzle or a more general set of rules in terms of their positioning.

The remainder of this paper is structured as follows. Firstly, the structure of the relay nozzle jet is considered and some points of attention concerning its modelling are discussed. Afterwards, the simulation and experimental setup employed in this research are introduced, alongside some considerations concerning the model. Two main cases are considered for simulation, one in which the relay nozzle exhausts into the free atmosphere ("free jet simulation") and one in which a yarn is inserted into the flow domain ("simulation with yarn"). The position and orientation of the inserted yarn are relevant for an air jet loom. This section is followed by a validation of the simulations based on a comparison of the simulation results with experimental data and scaling laws from the literature. Subsequently, the flow velocity and fluctuations obtained from the free jet simulation are analyzed. Finally, the transient forces experienced by the yarn are extracted from the simulation with yarn and compared to those estimated from an unsteady RANS (URANS) simulation. While with a URANS simulation forces due to e.g. vortex shedding can still be captured this type of simulation does not capture the turbulent eddies with a range of length scales present in the relay nozzle jet and consequently does not provide direct information about the forces that the weft 
experiences as a result of these eddies. Some additional explanation on RANS and LES modelling is provided in the Appendix in the section titled "The (dynamic) Smagorinsky model".

\section{The relay nozzle jet}

In air jet looms, the relay nozzles are supplied with highly pressurized air to obtain high-velocity jet flows. From a certain supply pressure onwards, the nozzle reaches its maximal volumetric flow rate and becomes choked. Often the supply pressure is increased beyond this point, imparting additional momentum to the jet and, thereby, increasing the force exerted by the relay nozzle jets on the yarn. However, this causes the pressure at the nozzle exit to exceed the pressure of the surroundings, which requires an expansion of the flow beyond the nozzle exit. The jet is then termed "underexpanded". ${ }^{23}$

Typically, relay nozzles are operated with a supply pressure of 3 to 5 bar. ${ }^{13}$ Several types of relay nozzles exist. A sketch of a relay nozzle in its simplest configuration is provided in Figure 1, which for the flow acts as a convergent nozzle.

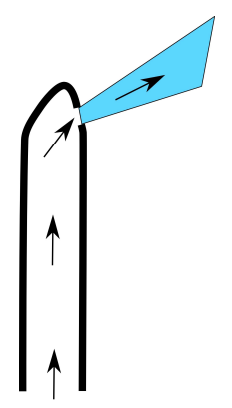

Figure 1. Sketch of a typical relay nozzle in its simplest configuration with an asymmetric outflow due to the bend.

In this research a convergent single-hole nozzle supplied with 5 bar absolute pressure is considered. Based on the work of Donaldson and Snedeker ${ }^{24}$ this will typically result in a "highly underexpanded jet" as the pressure ratio of the supply pressure to ambient pressure is larger than 3.846. The highly underexpanded character of the jet was indeed confirmed in the simulations. The typical "shock cells" can also be observed in the simulations of Adamek, Karel, Kolar et al. $^{25}$ and Osman ${ }^{26}$.

In this section some aspects of the highly underexpanded jet and its simulation using CFD are discussed because of their relevance to the current research. For a more in-depth discussion on underexpanded jets, the authors refer to the review paper of Franquet et al. ${ }^{21}$

Figure 2 shows the typical structure of a highly underexpanded jet.
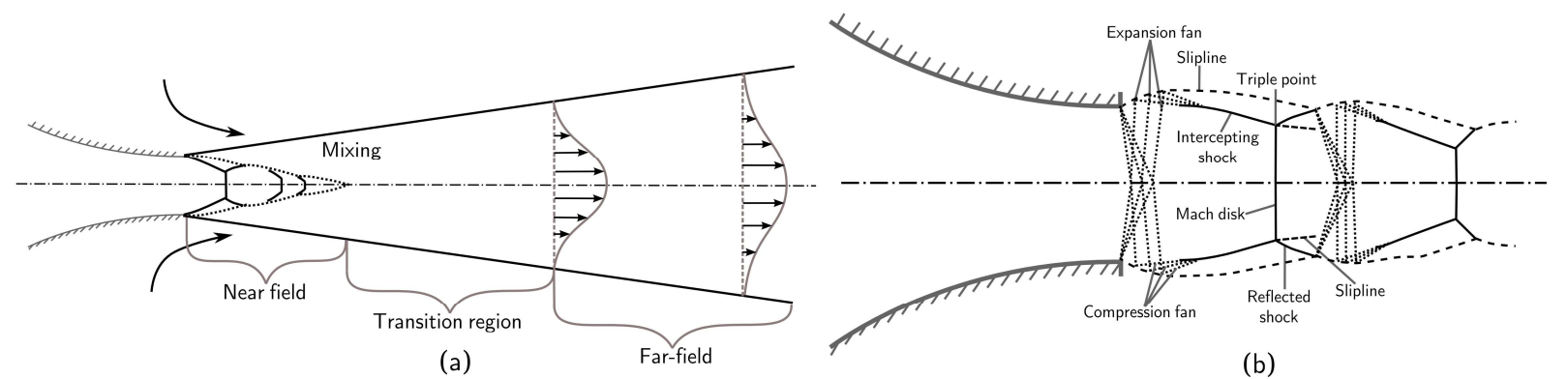

Figure 2. (a) Complete field of an underexpanded jet. (b) Near-field structure of a highly underexpanded jet. 
From Figure 2(b) it can be observed that the high supply pressure causes complex flow patterns at the nozzle exit consisting of shocks and expansion waves. Depending on the configuration, one or more shock cells with a barrel-like shape can occur. A highly underexpanded jet is characterized by the presence of a Mach disk (normal shock). ${ }^{21}$

As can be seen in Figure 2(a), the jet core is separated from the atmosphere by a mixing region. This region gradually grows in size until there is no distinct jet core visible anymore. This marks the start of the transition zone, where the distribution of the flow variables gradually transforms into that of a typical incompressible jet with self-similar profiles. ${ }^{21}$ Self-similarity in this case implies that the normalized radial profiles of the mean flow variables at different axial distances have the same distribution. The region where the jet is self-similar is referred to as the far-field.

Although the radial distribution of the flow variables in the far-field is the same as for an incompressible jet, the jet spreading and axial velocity decay can still be affected by the high supply pressure that was used. ${ }^{21}$ This is because the turbulent structures, responsible for mixing of the jet with the surroundings, are affected by the compressibility of the flow. Without taking these effects into account, a RANS simulation yields a too rapid decay of the jet velocity and therefore an inaccurate result, even in the far-field of the jet. ${ }^{26,27}$ Several models to include these effects exist (e.g. Wilcox ${ }^{27}, Z_{e m a n}{ }^{28}$, Sarkar, Erlebacher, Hussaini et al. ${ }^{29}$ ) but rely on empirical parameters, which can be case-dependent (e.g. different models/parameters for free shear flows and wall-adjacent flows). In this research the Wilcox model ${ }^{27}$ is used to include the compressibility effects for RANS simulations. However, to obtain the correct centerline decay rate the parameter " $\mathrm{M}_{\mathrm{t}}$ " has to be tuned. Unfortunately, this can adversely affect the calculated wall shear stress. ${ }^{27}$

\section{Methodology}

As mentioned in the introduction, in an air jet loom the interaction between the weft and the jet from the relay nozzle does not occur inside the nozzle or at the nozzle exit but rather some distance downstream of the nozzle exit (approximately 30 nozzle exit diameters) ${ }^{13}$. Under the current operating conditions this is located near the end of the transition zone/start of the far-field zone. This is confirmed by the self-similarity of the velocity profiles beyond this point, observed in both the experiment and simulation (depicted in Figure 8). This implies that there will be large scale turbulent structures present in the jet flow at the yarn location. These turbulent structures and their influence on the weft are the subject of this research.

\section{General framework and considerations}

To the authors' best knowledge no prior research has been performed regarding the turbulent vortices in a relay nozzle jet and their influence on the weft. Therefore, this research is aimed at establishing a rather general framework to provide an initial assessment and a starting point for future research instead of generating detailed, machine-specific data.

Because a relay nozzle has to enter in between the warp threads it can only take up a limited amount of space along the insertion direction. However, the relay nozzle jet has to be rather parallel to the insertion direction. Consequently, as can be seen in Figure 1, the flow direction changes rather abruptly close to the nozzle exit, which causes asymmetry in the jet and a dependence of the jet direction on the supply pressure. Therefore, the use of a real relay nozzle geometry in this research would complicate not only the setup of the model but also the measurements required for validation. Therefore, in this research, a more general geometry was opted for, in which the jet is symmetrical and its direction independent of supply pressure. A sketch of the nozzle used for the experiments is provided in Figure 3. Due to the use of a large radius of curvature in the nozzle, only the section of the nozzle highlighted in green had to be included in the simulation. In addition, with regards to future testing, this relay nozzle geometry allows for an easier and 
more accurate positioning and orientation of the relay nozzle jets with respect to the reed (as long as the warp threads and/or beat-up motion can be omitted). By omitting the asymmetry in the jet the results of the simulations will deviate from the real situation. However, this asymmetry will not prevent the origination nor the growth of turbulent vortices in the relay nozzle jet and therefore not invalidate the general observations in this research. Additionally, the simulation methodology, mesh sizes and time step sizes described in this research can also be applied to other single-holed relay nozzle geometries provided the mesh is aligned appropriately.

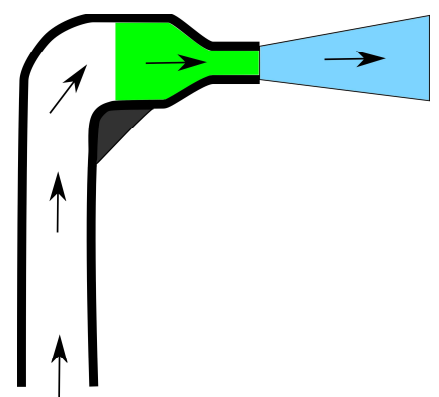

Figure 3. Sketch of the test nozzle geometry with a symmetrical outflow.

Another side note that has to be made is that in the current research the presence of the reed is omitted as including this into an LES would result in an even higher computational cost due to the increased mesh requirements. The reed will affect the turbulent vortices and the interaction between the relay nozzle flow and the weft as that is its main purpose. However, turbulent vortices will definitely still be present in the reed channel. In order to study their behavior and interaction with the weft it is of course mandatory that the jet itself and its turbulence can be simulated appropriately. This paper is aimed at doing just that.

The relay nozzle configuration selected for this investigation had a diameter of $1.6 \mathrm{~mm}$ and was supplied with a pressure of 4 barg (bar gauge). For the remainder of this paper, the nozzle exit diameter will be referred to as " $D$ ". Initially, a simulation was performed with the jet exhausting into the free atmosphere to evaluate the selected LES methodology and to extract information about velocity fluctuations. Afterwards, a straight, rigid cylinder was inserted into the domain to represent a yarn and the forces exerted on this cylinder were analyzed.

\section{Numerical model}

To resolve the aforementioned turbulent structures an LES methodology is required. Additionally, since in an LES the larger turbulent structures are resolved, the effects of compressibility on these structures can be captured without additional modelling. The modelling on the small scales will have relatively limited effect on the mixing. Consequently, contrary to simulations with a RANS model, there was no need to tune any parameters to properly capture the effect of compressibility on the mixing with the selected LES methodology. As stated by Yoder, DeBonis and Georgiadis ${ }^{30}$, LES models have shown to be effective at modelling free shear flows.

Geometry and boundary conditions.

A sketch of the flow domain without the yarn is displayed in Figure 4. The figure also shows a cross section of the mesh at the nozzle exit. 


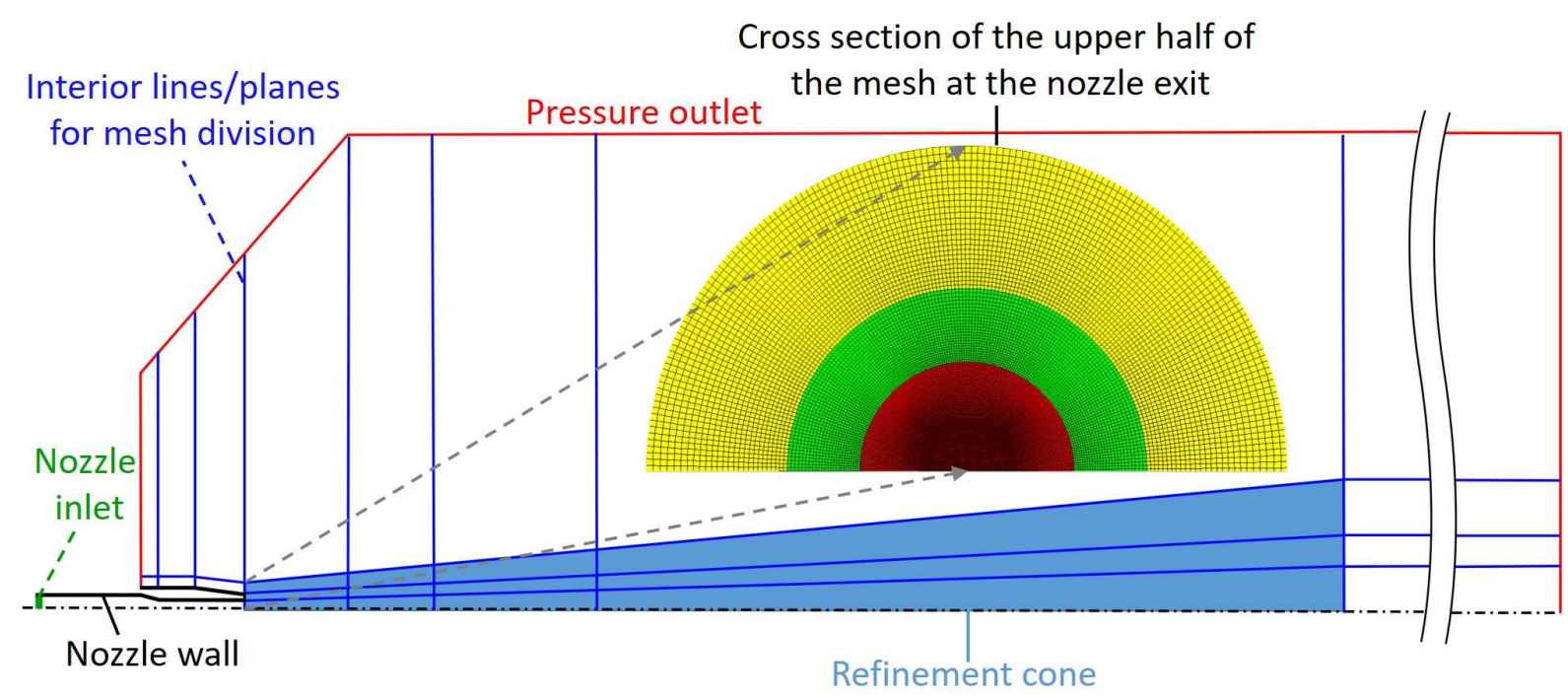

Figure 4. Sketch of the flow domain including a cross section of the mesh at the nozzle exit.

Referring to Figure 4, the boundary conditions for the CFD-problem are as follows: the thick green line on the left-hand side represents a pressure inlet where a total pressure of 4 barg is imposed. The solid black lines indicate the walls of the nozzle where neither slip nor heat transfer is allowed. The solid blue lines are interior lines used for generation of a block-structured grid and do not affect the simulation. The solid red lines are specified as pressure outlets where an absolute static pressure of $100000 \mathrm{~Pa}$ is imposed.

The flow domain extends up to 120D downstream of the nozzle exit and 30D radially outwards from the nozzle axis. The inlet diameter of the nozzle is $1.56 \mathrm{D}$. As can be seen from Figure 4, the aforementioned blocks form an expanding cone (blue region in Figure 4) starting at the nozzle exit with a diameter of 3D. The slope of the cone was chosen according to the expected expansion ratio of the jet as predicted by Yüceil et al. ${ }^{31}$ and extends up to 70D downstream of the nozzle exit. Additional details on the mesh will be provided in the section titled "Mesh resolution and quality".

\section{Simulation settings and procedure.}

In this research the unsteady behavior of the jet after it has fully developed was of interest (often referred to as "the quasi-steady-state behavior"). Starting the LES from a zero-flow field would waste a lot of computational time simply to progress the simulation in time up to the quasi-steady state which does not yield relevant data for the study at hand. Therefore, a steady-state RANS simulation was performed first and the solution was used as initial condition for the LES. By doing so, the LES can be started from a reasonably good estimate of the fully developed jet (in which the turbulent eddies were averaged-out) and a quasi-steady-state can be reached with less computational time. The choice of initial condition will not influence the final result because the quasi-steady-state was of interest and a sufficiently long calculation time was used. All simulations in this research were performed with ANSYS Fluent 18.1. The air was considered to obey the ideal gas law $\left(p=\rho R T\right.$, with $\left.R=287 \frac{\mathrm{J}}{\mathrm{kg} \cdot \mathrm{K}}\right)$.

The steady-state RANS simulation was performed using the k- $\omega$ SST model. To obtain an adequate initial flow field, the compressibility effects had to be taken into account. To this end, Wilcox's model ${ }^{27}$ was used in the current research. Based on comparison with experimental data, the parameter $\mathrm{M}_{\mathrm{t} 0}$ was set to 0.1 instead of the default 0.25 . 
For the LES, the dynamic Smagorinsky model was employed since dynamic models have shown to better reproduce the behavior of underexpanded jets compared to eddy viscosity models such as the original Smagorinsky model. ${ }^{30,32}$ The coupled scheme was used for the pressure-velocity coupling. A bounded second-order implicit time stepping scheme was used with a time step size of 3.5e-08 s. The solver was allowed to perform 10 iterations per time step, typically yielding a residual drop of 3-to-4 orders of magnitude with tolerances set to $1 \mathrm{e}-6$ for the continuity, momentum and energy equations. For the convective terms in the flow equations, a second-order upwind scheme was employed for the energy and a bounded-central differencing scheme for density and momentum. For the reader's convenience some additional explanation on LES and more specifically the (dynamic) Smagorinsky model is provided in the Appendix in the section titled "the (dynamic) Smagorisnky model".

\section{Mesh resolution and quality.}

In LES a sufficiently fine, high quality grid is required. Additionally, for highly underexpanded jets the mesh in the near-field needs to be extra fine due to the presence of shocks and expansion waves. Typical mesh refinement studies are very resource intensive and not as straightforward because LES is by definition grid-dependent and time-sensitive. Therefore, mesh resolutions are often selected and evaluated based on comparison with experiments, data from literature and LES-specific quality criteria. ${ }^{33}$

To the authors' best knowledge, LES specifically for relay nozzles in looms has not yet been performed. But, for highly underexpanded jets in general, there have been some LES studies. These can be used as a starting point for the current simulations. For example, Vuorinen, Yu, Tirunagari et al. ${ }^{34}$ employed implicit LES on highly underexpanded air jets to study the mixing for direct injection gas engines. Hamzehloo and Aleiferis ${ }^{35}$ also performed research related to direct injection gas engines by doing LES using the WALEmodel for hydrogen jets. Li, Zhou, Yao et al. ${ }^{36}$ studied the influence of nozzle shape on the mixing and penetration rate of highly underexpanded nitrogen jets for different nozzle geometries in light of passive flow control applications. Additional details about the mesh, supply pressures and zones of interest for the aforementioned LES-research are provided in Table 1. The "zone of interest" corresponds to the region subject to investigation where the quality and refinement of the mesh were selected to obtain good LES results. Notice that the axial extent of these zones is quite limited as these investigations focus more on the near-field behavior. LES research into the far-field of highly underexpanded jets is very scarce. Most likely, due to the increase in computational cost when extending the domain downstream and because the number of applications in which time-accurate data in the far-field is important is relatively limited. However, as mentioned in the section titled "The relay nozzle jet" the main interaction between the jet from the relay nozzle and the yarn usually happens about 30 diameters downstream of the nozzle. This typically corresponds to the end of the transition zone/beginning of the far field, which is also confirmed by the selfsimilarity of the velocity profiles as illustrated in Figure 8. In recent years LES modelling has also been applied to highly underexpanded hydrogen jets with regards to hydrogen safety ${ }^{36-38}$. 
Table 1. Additional case details for previously mentioned LES research on underexpanded jets. $(A=$ axial, $R=$ radial $)$

\begin{tabular}{|c|c|c|c|c|}
\hline Source & Case specifics & $\begin{array}{c}\text { Supply pressure(s) } \\
\text { [barg] }\end{array}$ & $\begin{array}{l}\text { Zone of } \\
\text { interest }\end{array}$ & $\begin{array}{c}\text { Near-nozzle grid } \\
\text { spacing }\end{array}$ \\
\hline Vuorinen et al. ${ }^{34}$ & DI gas engines & $\begin{array}{l}3.5,4.5,5.5,6.5 \\
7.5\end{array}$ & $\begin{array}{l}\text { A: } 22.5 \mathrm{D} \\
\mathrm{R}: 3.0 \mathrm{D}\end{array}$ & $\begin{array}{l}\text { A: D/35 } \\
\text { R: D/70 }\end{array}$ \\
\hline $\begin{array}{l}\text { Hamzehloo and } \\
\text { Aleiferis }^{35}\end{array}$ & DI gas engines & $8.5,10,30,70$ & $\begin{array}{l}\text { A: } \quad 20 \mathrm{D} \\
\mathrm{R}: \quad 8 \mathrm{D}\end{array}$ & $\begin{array}{l}\text { A: } D / 50 \\
\text { R: } D / 50\end{array}$ \\
\hline $\mathrm{Li}$ et al. ${ }^{36}$ & Passive flow control & 5.6 & $\begin{array}{ll}\text { A: } & 40 \mathrm{D} \\
\mathrm{R}: & 25 \mathrm{D}\end{array}$ & $\begin{array}{l}\text { A: D/67 - D/25 } \\
\text { R: D/200 - D/52 }\end{array}$ \\
\hline
\end{tabular}

The mesh used in the current research was chosen to closely resemble that of Vuorinen et al. ${ }^{34}$ as the configurations are similar. However, the zone of interest/refinement zone for the current research extends further downstream (up to 70D) to adequately capture the interaction with the yarn. Within the cone, expansion ratios were selected as to keep the aspect ratio of the cells close to 1 . The circumferential direction is meshed with 312 equally spaced cells. At the nozzle exit the smallest cells have a radial spacing of $\mathrm{D} / 100$ (increasing to $\mathrm{D} / 30$ at the outer boundary of the cone) and an axial spacing of $\mathrm{D} / 35$. At a distance of 70D from the nozzle exit the largest cells within the cone have an axial spacing of $\mathrm{D} / 17$, a radial spacing of $\mathrm{D} / 12$ and a circumferential spacing of $\mathrm{D} / 14$. Outside of the cone the cells are allowed to expand quite rapidly (cell-to-cell ratio of 1.05 for the radial dimension and 1.1 for the axial dimension). In total the mesh contains approximately 47 million hexahedral cells.

Several LES quality criteria have been evaluated: ratio of turbulent kinetic energy, relative effective viscosity index, turbulent energy spectra and two-point correlations. Additional explanation on these criteria and the results for the case at hand are provided in the Appendix in the section titled "LES quality criteria”. All 4 quality criteria evaluated satisfactorily within the refinement region.

Inclusion of the yarn.

From the simulations without yarn, information about the velocity in the vicinity of the yarn can be obtained. By using force coefficients a yarn force can then be estimated. However, the use of force coefficients is often not accurate enough when considering local forces and time-varying flow fields. To obtain more accurate forces, a rigid cylinder (representing a yarn) is introduced in the simulation. This cylinder has a diameter of $0.72 \mathrm{~mm}$ and a smooth outer surface. For convenience the $\mathrm{x}$-axis in this simulation was aligned with the yarn-axis so that lengthwise and lateral forces could easily be separated. By contrast, in the simulation without yarn, the $\mathrm{x}$-axis was aligned with the nozzle axis.

The position and orientation of the cylinder was chosen to be representative of a yarn located in the reed channel of an air jet loom. This implies that the yarn is not parallel to the axis of the jet. Therefore, the (test) nozzle is positioned in such a way that its jet attacks the yarn at a similar angle as the jet from a real relay nozzle in an air jet loom. However, this makes the construction of a high quality conformal grid with an acceptable number of cells practically very difficult. Therefore, in this research, it was decided to make adaptations to the previously constructed mesh. In order to do so a Python code was written to create a cylindrical hole in the flow domain with a radius exceeding the yarn radius. This cylindrical hole is obtained by selectively removing cells and subsequently projecting the newly created outer cell faces onto a cylindrical surface. Afterwards, a high-quality, hexahedral, cylindrical-shaped grid (referred to as the yarn mesh from here on) which includes the yarn is inserted into the created cavity. The outer faces of the yarn mesh are then configured as a non-conformal interface with the rest of the flow domain. The resulting configuration is illustrated in Figure 5 with detailed views of the newly created interface. The y-axis points vertically upwards and the z-axis points away from the yarn towards the nozzle. As is shown in the figure, 
the choice was made not to extend the yarn throughout the entire domain as this would reduce the quality of the interface due to the stretching of the cells towards the ends of the domain. On the left side of the figure a sketch is provided to illustrate the position of the components in the loom. The reed is not part of the simulation model.

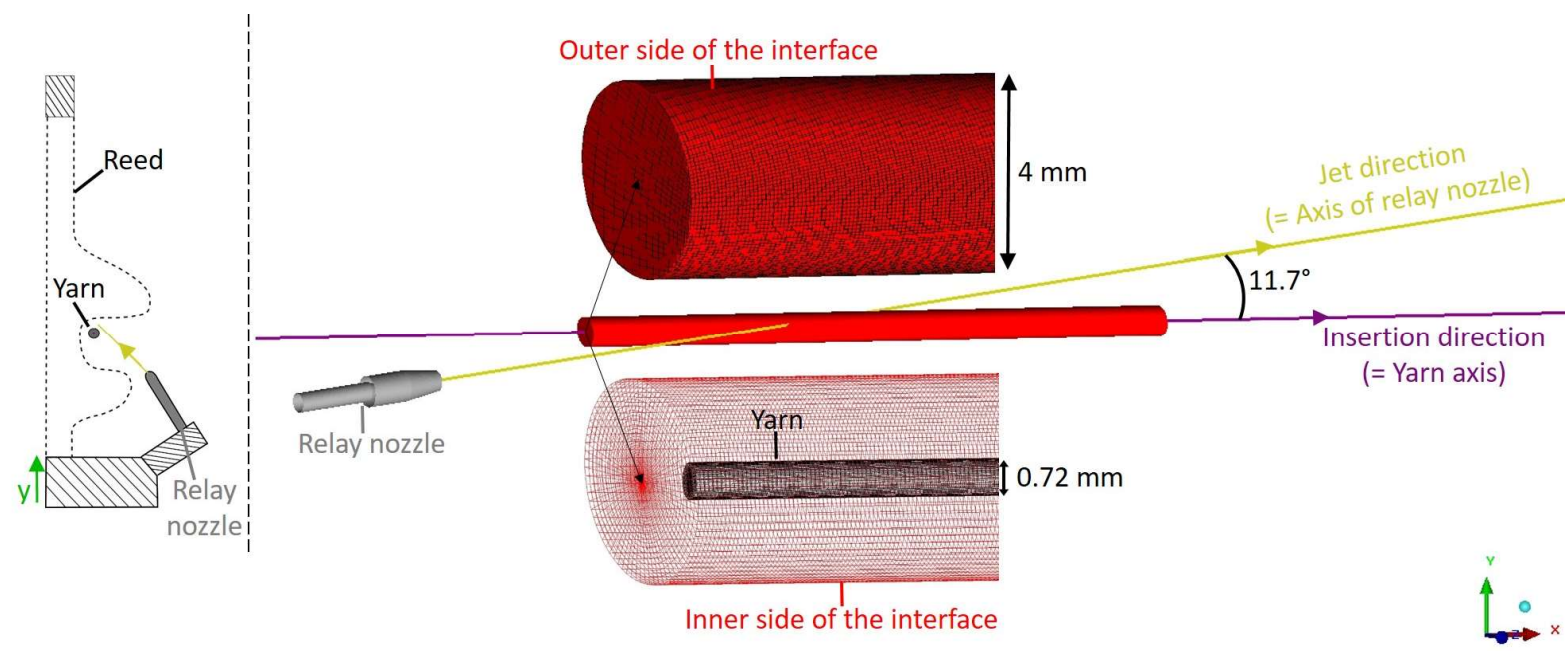

Figure 5. Position of the components in a loom, with the reed indicated using a dotted line because it is not included in the presented simulations (left). Relative position of the yarn with respect to the relay nozzle, with details of the mesh on the outer and inner side of the interface around the yarn (right).

For the yarn a radius of $0.36 \mathrm{~mm}$ was chosen, the interface had a radius of $2 \mathrm{~mm}$. Additional information on the geometry (for the simulations with and without yarn) is provided in Table 2. From the table it can be seen that for the simulation with yarn, the nozzle exit was chosen as origin and the yarn axis was parallel to the $\mathrm{x}$-axis of the coordinate system. For the simulation without yarn, the post-processing is performed on a line with the same relative orientation and position with respect to the nozzle as the yarn centerline in the simulation with yarn. The start and end point of this line are also included in the table.

Table 2. Additional information on the geometries provided in vector form.

\begin{tabular}{|c|c|c|c|}
\hline \multicolumn{2}{|c|}{ Geometry with yarn } & \multicolumn{2}{|c|}{ Geometry without yarn } \\
\hline Geometrical object & {$[\mathbf{X}, \mathbf{Y}, \mathbf{Z}]$} & Geometrical object & {$[\mathbf{X}, \mathbf{Y}, \mathbf{Z}]$} \\
\hline Yarn start point & {$[16.3,3.1,-6.9] \cdot D$} & Line start point & {$[20.0,1.2,6.9] \cdot D$} \\
\hline Yarn end point & {$[63.8,3.1,-6.9] \cdot D$} & Line end point & {$[91.8,-6.9,19.4] \cdot D$} \\
\hline Center of nozzle exit & {$[0.0,0.0,0.0]$} & Center of nozzle exit & {$[0.0,0.0,0.0]$} \\
\hline Nozzle direction vector & {$[0.98,0.11,-0.17]$} & Nozzle direction vector & {$[1.0,0.0,0.0]$} \\
\hline
\end{tabular}

\section{Experimental setup}

By means of pitot measurements, experimental data was collected for the free jet flow of the test nozzle (sketched in Figure 3). The pitot probe was positioned robotically with reference to the exit of the relay nozzle. The total pressure was measured in planes perpendicular to the jet axis at axial distances of 20D, 30D, 45D, 60D and 75D. In each plane measurements were performed at $2401(49 \mathrm{x} 49)$ locations, which were equally spaced. The outer boundaries of the measurement plane were chosen based on the axial distance to the nozzle. This data is summarized in Table 3. 
The pitot probe had an inner diameter of $0.3 \mathrm{~mm}$ and an outer diameter of $0.51 \mathrm{~mm}$. To be able to cover the expected pressure range with good accuracy, the pitot probe was connected to 3 pressure sensors (measurement range: $0-100$ mbar, $0-400$ mbar and $0-2.5$ bar). The probe was kept stationary at each measurement location for 2.1 seconds. During the first 2.0 seconds no measurements were performed as the air in the pitot probe has to adapt to its new position. Afterwards, the pressure was sampled for 0.1 seconds with a sample rate of $100 \mathrm{kHz}$, which yielded 10000 samples. For each measurement location, the software selected and stored the average pressure from the most appropriate sensor (based on the observed pressure level).

The supply pressure for the relay nozzle was stabilized by an automatic pressure regulator. From the measured total pressure $\left(p_{\text {tot }}\right)$ the velocity was calculated based on Formula $(1)^{39}$, where it is assumed that the axial locations are sufficiently far downstream so that the total temperature $\left(\mathrm{T}_{0}\right)$ and static pressure $(p)$ are uniform throughout the jet and equal to $293 \mathrm{~K}$ and atmospheric pressure, respectively. Formula (1) should not be used for supersonic flows as then a bow shock forms in front of the pitot probe, which is not an isentropic process.

$\mathrm{v}=\sqrt{2 \cdot \frac{\gamma}{\gamma-1} \cdot R \cdot T_{\text {tot }} \cdot\left[1-\left(\frac{p}{p_{\text {tot }}}\right)^{\frac{\gamma}{\gamma-1}}\right]}$

Table 3. Summary of measurement positions.

\begin{tabular}{|r|r|r|}
\hline Axial distance & $\begin{array}{c}\text { Width and height of } \\
\text { measurement plane [mm] }\end{array}$ & \multicolumn{2}{|c|}{ Resolution [mm] } \\
\hline $20 \mathrm{D}$ & 10.80 & 0.225 \\
\hline $30 \mathrm{D}$ & 16.08 & 0.335 \\
\hline $45 \mathrm{D}$ & 24.00 & 0.500 \\
\hline $60 \mathrm{D}$ & 31.92 & 0.665 \\
\hline $75 \mathrm{D}$ & 39.84 & 0.830 \\
\hline
\end{tabular}

\section{Results}

\section{Validation}

Before analyzing the simulation results it is essential that some verification of the model is performed. In order to do so, simulations are performed for a jet exhausting into the free atmosphere and the calculated centerline velocity and jet spreading are compared to experimental data and data from literature.

In Figure 6 the centerline velocity as obtained from different CFD simulations is compared to the experimental data. 


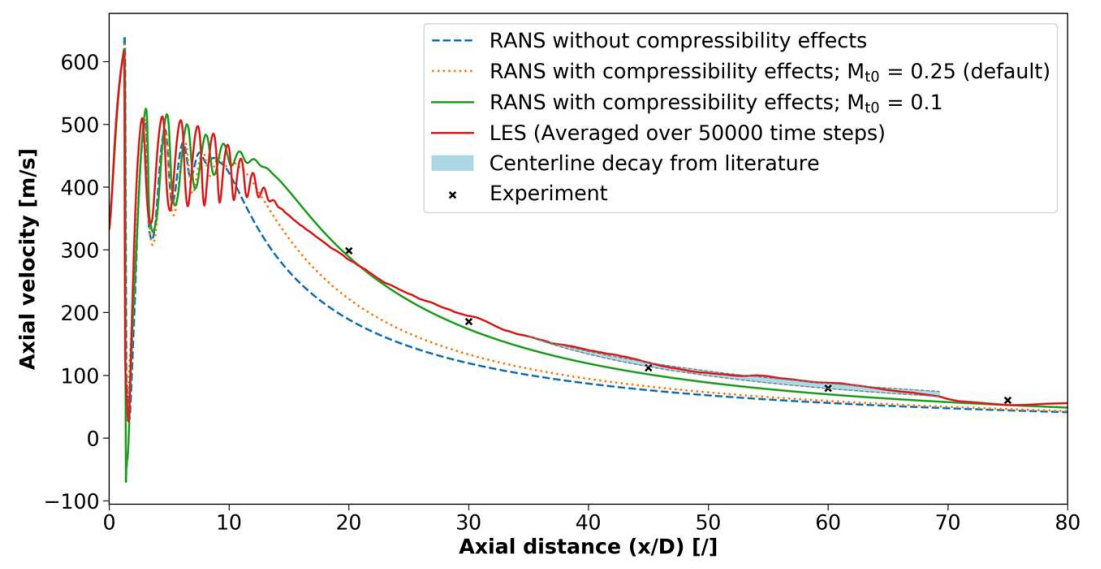

Figure 6. Axial velocity on the centerline of the free jet as obtained from simulations compared to experimental data.

From the figure it can be observed that it is indeed essential to take into account the compressibility effects in the RANS simulation to obtain an adequate decay rate. Furthermore, the parameter $\mathrm{M}_{\mathrm{t} 0}$ needs to be tuned. On the other hand, the mean centerline velocity obtained from the LES shows good correspondence with the experimental data without any tuning. The figure also provides a comparison to the scaling law as proposed in Yüceill et al. ${ }^{31}$ : the small zone colored in light blue starts from the simulated LES curve at 35D and extends downstream according to the proposed scaling law with a $\pm 10 \%$ tolerance on the spreading rate. The correspondence is rather good.

To obtain time-averaged data from the LES the flow was averaged over 90 flow-through times after an initial 140 flow-through times were simulated. The "flow-through time" is defined as the time required for a particle to travel from the nozzle exit to the end of the domain when it follows the centerline velocity as calculated from the RANS simulation with tuned $\mathrm{M}_{\mathrm{t} 0}$.

From here on, simulation data always refers to LES data. Aside from a comparison of the centerline velocity decay, the velocity profiles in several orthogonal planes (perpendicular to the jet axis) can also be compared. This comparison is shown in Figure 7, where the profiles are obtained by circumferential averaging of the data.

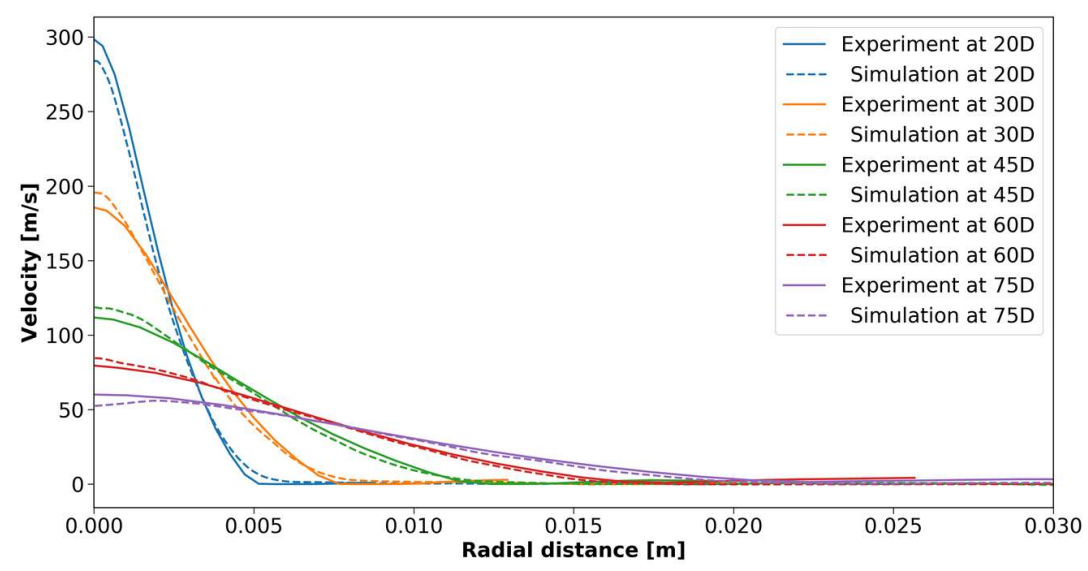

Figure 7. Comparison of velocity profiles in several orthogonal planes. 
In Figure 7 some minor discrepancies can be observed but the overall agreement is good. As was mentioned previously, in the far-field the radial profiles should become self-similar if a proper nondimensionalization is applied; this is illustrated in Figure 8 for the experimental and LES data. In this figure $\mathrm{v}_{\mathrm{CL}}$ is the centerline velocity and $b_{v}$ is the jet half-width (radial distance at which the velocity equals $1 / 2$ of the centerline velocity at that axial distance). The collapse of the curves for both simulation and experiment is satisfactory apart from the LES data at 75D. However, this curve is located outside of the refinement region.

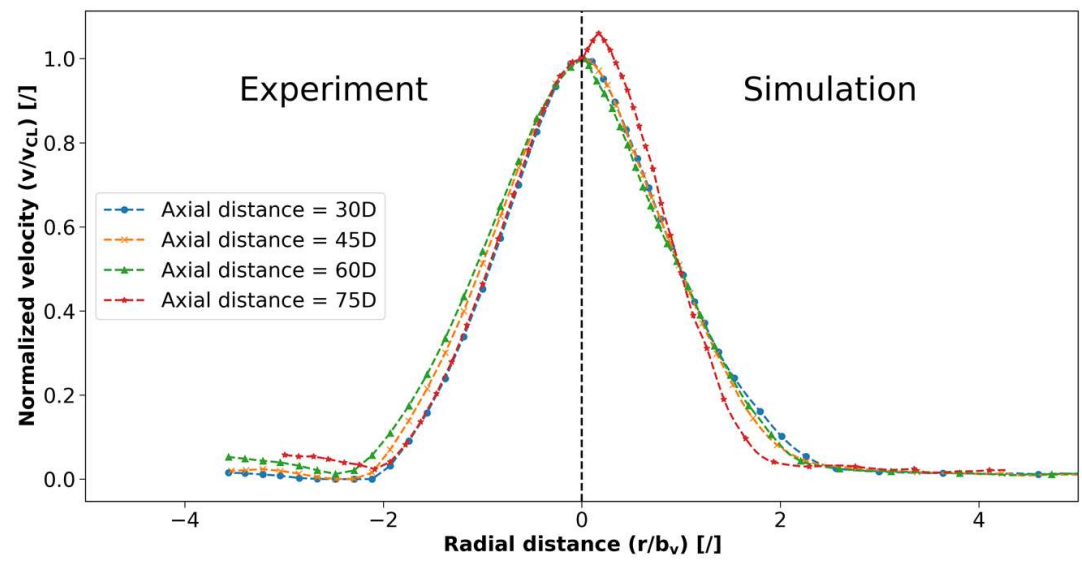

Figure 8. Nondimensionalized radial velocity profiles for the experimental data (left) and simulation data (right).

\section{Analysis of velocity fluctuations without yarn}

In Figure 9 the simulated velocity field is visualized. The central image, which shows the instantaneous velocity magnitude, contains a line indicating the considered yarn location relative to the nozzle. Note that for the free-jet case the x-axis is aligned with the nozzle axis. The dotted line indicates that the yarn is behind the visualization plane. 

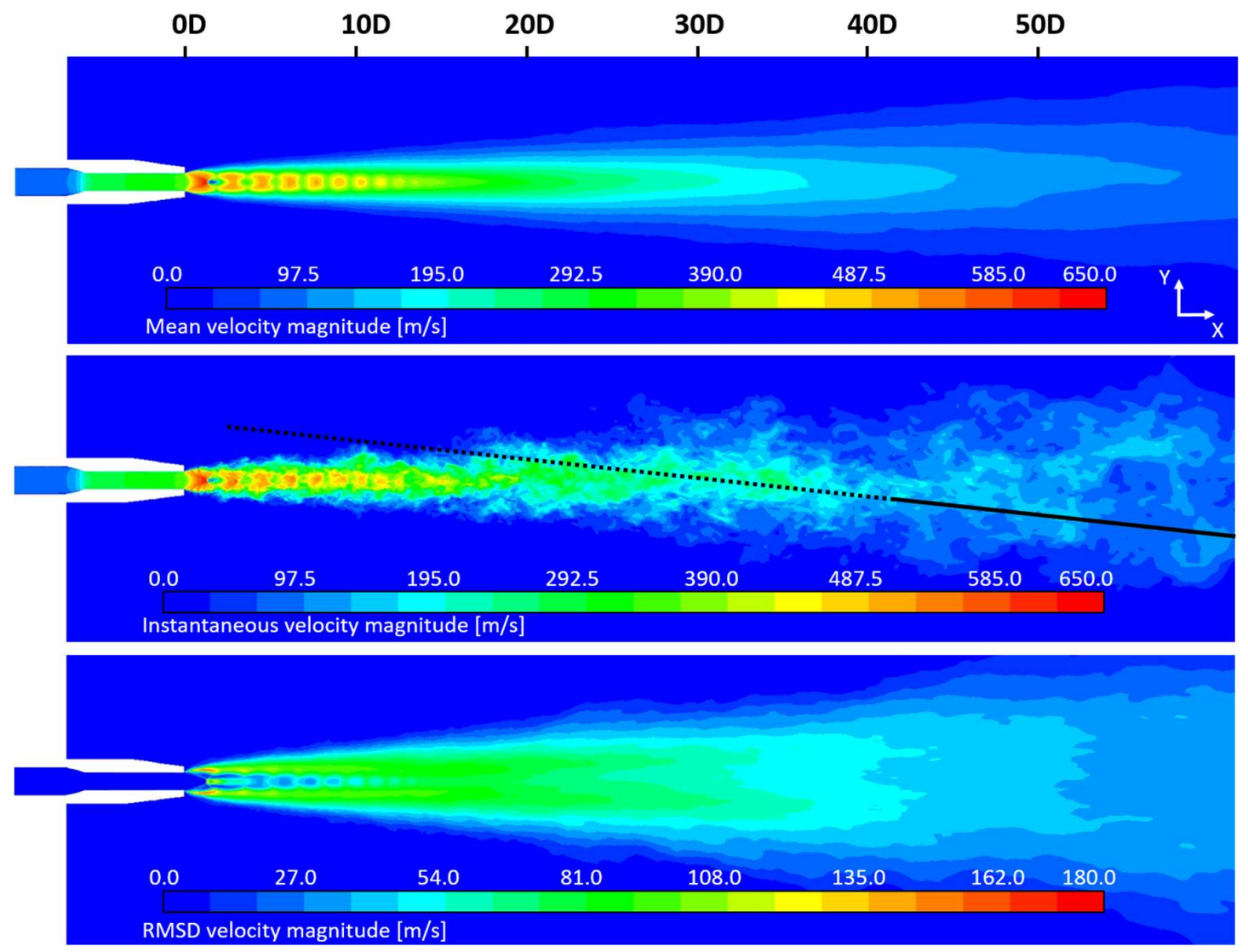

Figure 9. Simulated velocity magnitude and its fluctuation for the relay nozzle jet.

From Figure 9 several observations can be made. First of all, it is apparent that there is a huge difference between the mean velocity field (as one would obtain from RANS) compared to the instantaneous velocity field. Secondly, the root-mean-squared deviation (RMSD) clearly shows that while the velocity in the nozzle itself is rather constant in time, large velocity fluctuations quickly originate outside of the nozzle. These oscillations mainly originate from the shear layer and an unsteadiness in the shock pattern. Thirdly, from the instantaneous velocity magnitude it can be observed that the yarn crosses the jet in an area with large-scale vorticial structures. One can also note that the figures of mean and RMSD velocity are not completely symmetrical (yet) far from the nozzle (i.e. for $\mathrm{x}>35 \mathrm{D}$ ). This implies that the averaging should be continued further in time to obtain more accurate flow-averaged values in the far away regions as there is no inherent reason for the mean flow to be asymmetric. However, getting the simulation up to this point already required 43.5 days of computational time on 840 cores $(30$ nodes, each node having 2 Xeon E5$2680 \mathrm{v} 4$ processors of $2.4 \mathrm{GHz}$ with 14 cores per processor). Obtaining a perfectly symmetrical averaged flow field even more distant from the nozzle would further increase the computational time, while having little added value for the current investigation.

The relative importance of the velocity fluctuations with respect to the mean velocity magnitude can be visualized by plotting the ratio of the RMSD of the velocity magnitude to its mean value. This is presented in Figure 10. At the right bottom of this figure the ratio is visualized in a cross section by means of a contour plot, the vertical lines indicate the orthogonal planes used for generating the graph. The corresponding RMSD values are displayed in Figure 11. 


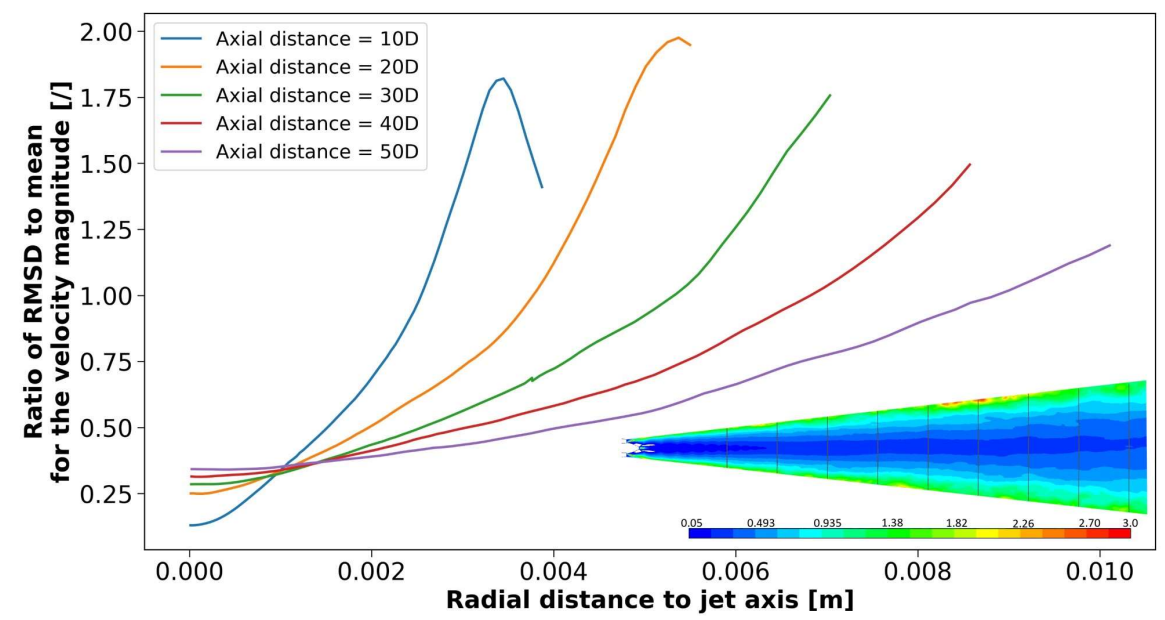

Figure 10. Circumferentially averaged ratio of the RMSD of the velocity magnitude to its mean value in several orthogonal planes.

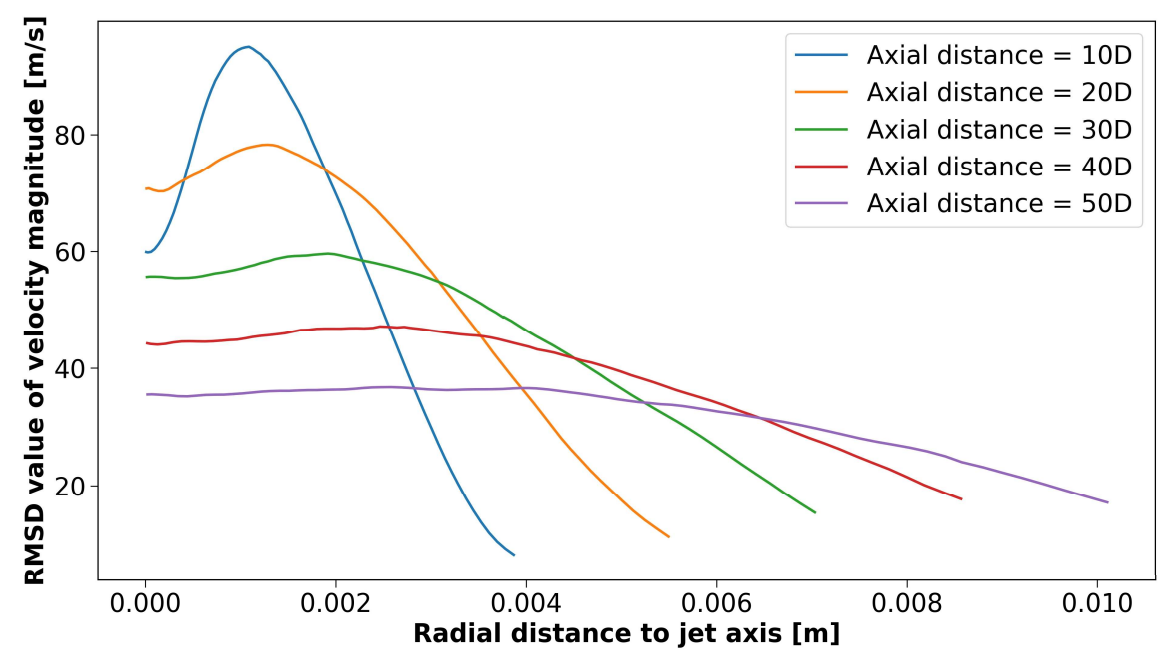

Figure 11. Circumferentially averaged RMSD values of velocity magnitude in several orthogonal planes.

From Figure 10 it is quite apparent that the relative importance of the fluctuations decreases as the radial distance to the jet axis decreases except at relatively large distances from the jet axis, where the mean velocity magnitude becomes very low, there the ratio also decreases with increasing radial distance. This implies that, to reduce the relative influence of turbulent fluctuations on the weft while maintaining. a high propulsive force, it would be desired to align the weft with the jet centerline as well as possible, implying that the nozzle should be positioned as closely as possible to the weft and with its jet aligned with the insertion direction as well as possible. Furthermore, it can be noticed from Figure 11 that up to 20D there is a distinct drop in RMSD value towards the jet centerline due to the presence of the jet core. However, further downstream the RMSD value tends to become uniform throughout the jet as it becomes dominated by turbulent structures. One way of extending the jet core and thereby the region in which turbulent fluctuations are relatively less important, is by further increasing the supply pressure of the relay nozzles.

Another interesting way of looking at the simulation results is by investigating the velocity information along a line representative for the weft in an air jet loom. In Figure 12 the velocity information along the line presented in Figure 9 is plotted. The starting point of the yarn was chosen to be at an orthogonal distance 
from the nozzle exit of 20D. Additionally, Figure 13 shows the axial velocity as a function of time in a point located at 47D from the nozzle exit, close to the hypothetical yarn.

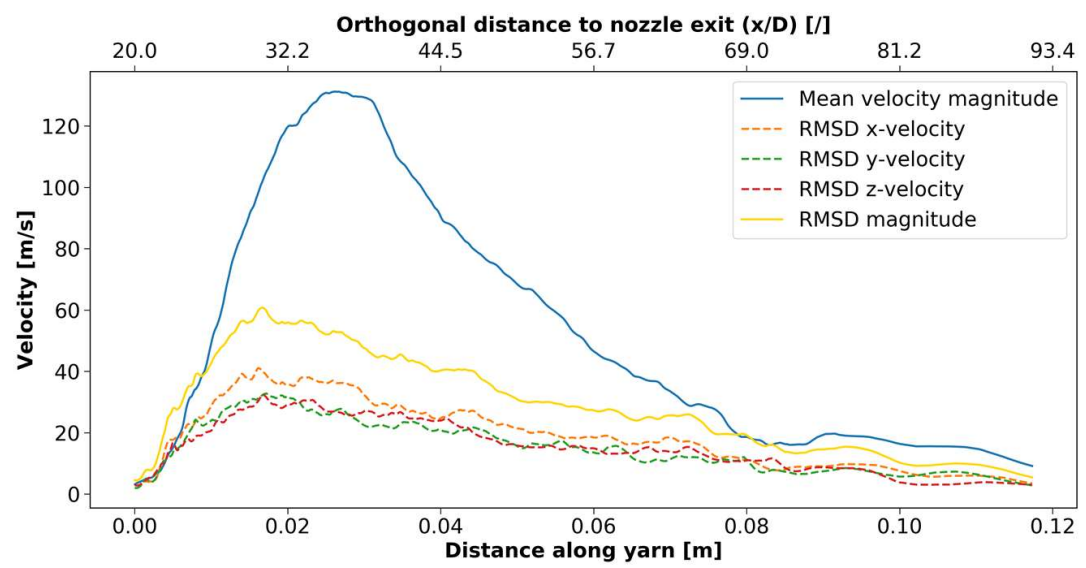

Figure 12. Mean and RMSD of the velocity magnitude at the yarn location.

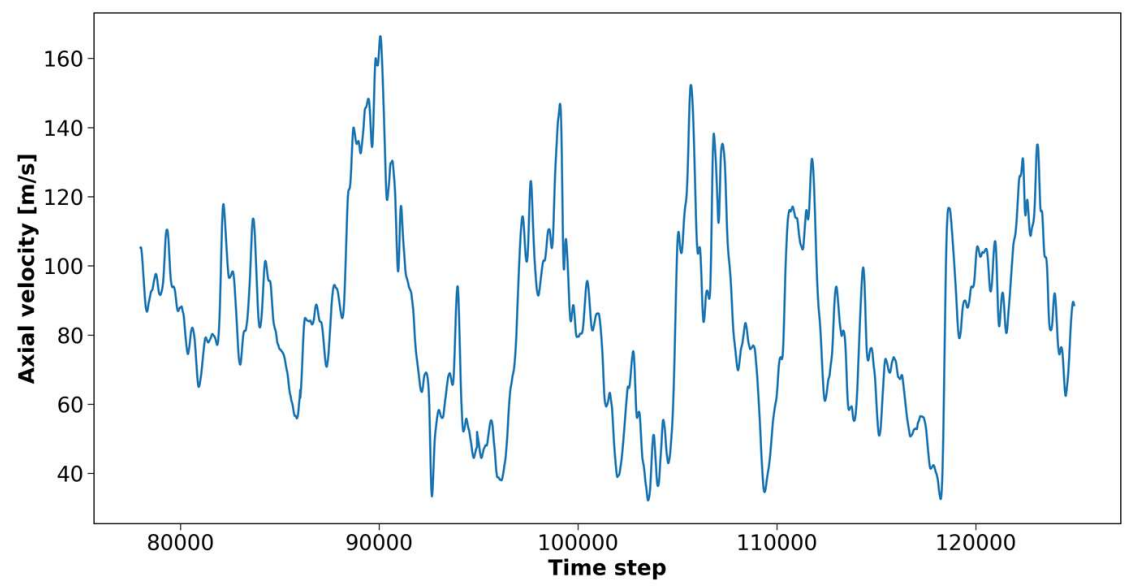

Figure 13. Axial velocity as a function of time in a point located at [47D, 2.25D, 0.0].

In Figure 12 it can be observed that along the weft the velocity fluctuations can become relatively large compared to the mean velocity magnitude. In the high velocity region, where the largest forces will be transferred to the yarn, the fluctuations are in the order of $50 \%$ of the mean velocity magnitude. As can be seen in Figure 12, the magnitude of the fluctuations is nearly the same in the $x-, y$ - and z-directions, with those in the $\mathrm{x}$-direction being slightly larger.

Because the relay nozzle jet is oriented rather parallel to the insertion direction (in this case at an angle of $11.7^{\circ}$ ), the mean transversal velocity component is small (approximately $1 / 5^{\text {th }}$ of the velocity magnitude). This implies that the turbulent velocity fluctuations are quite substantial compared to the mean transversal velocity magnitude and should therefore not be neglected concerning the transversal motion of the weft without further investigation. Especially close to the tip of the weft, where the tension in the yarn is limited, the turbulent oscillations can have a substantial influence.

To complete this section, an estimate of the forces on a yarn can be obtained from the velocity field by using a force coefficient. Osman et al ${ }^{18}$ reported a longitudinal force coefficient of $7 \mathrm{e}-05$ and an orthogonal force coefficient of 7.64e-04 for a cotton yarn of 104 tex. Using these force coefficients, the fluctuation in 
the y- and z-direction would reach up to approximately $0.47 \mathrm{~N} / \mathrm{m}$ with, at that location, an $\mathrm{x}$-force of approximately $0.55 \mathrm{~N} / \mathrm{m}$. In conclusion, the force fluctuation is about $80 \%$ of the force magnitude based on the velocity data and force coefficients. This analysis is for a stationary weft. For a weft that is travelling at a certain velocity in the weft direction, the relative velocity of the air with respect to the weft should be used in the formula. This will reduce the component of the force along the weft direction while leaving the transversal forces almost unaffected, thereby making them even more prominent.

\section{Analysis of force fluctuations on yarn}

More accurate data about the forces experienced by a yarn can be obtained by inserting a cylinder into the flow domain and calculating the pressure and shear forces it experiences. In a loom the yarn is not aligned with the relay nozzle axis, which makes the practical aspect more difficult. Details about the implementation can be found in the section "Inclusion of the yarn". Due to the interpolation of flow variables at the nonconformal interface (see Figure 5) some error is introduced into the simulation. Therefore, it is important to verify that the interface does not drastically disturb important flow features.

Similar to the analysis without yarn, a steady-state RANS simulation is executed first to obtain an initial flow field for the LES. Additionally, the output of the steady-state RANS simulation is also used as initial flow field for an unsteady RANS (URANS) simulation with the same time step size as the LES to provide a more fair comparison. Bear in mind that, compared to the simulation without yarn, the $\mathrm{x}$-axis is now aligned with the yarn-axis instead of the nozzle-axis.

In Figure 14 the velocity field is plotted for both the URANS simulation and the LES. The top figures show the mean velocity magnitude in a plane with normal vector $[-0.20,0.69,-0.69]$ and containing the nozzle axis. The bottom 2 figures plot the instantaneous velocity in a plane perpendicular to the z-axis and containing the yarn-axis. Visually, there is no difference between the mean and instantaneous velocity for the URANS simulation as the fluctuations are negligibly small.

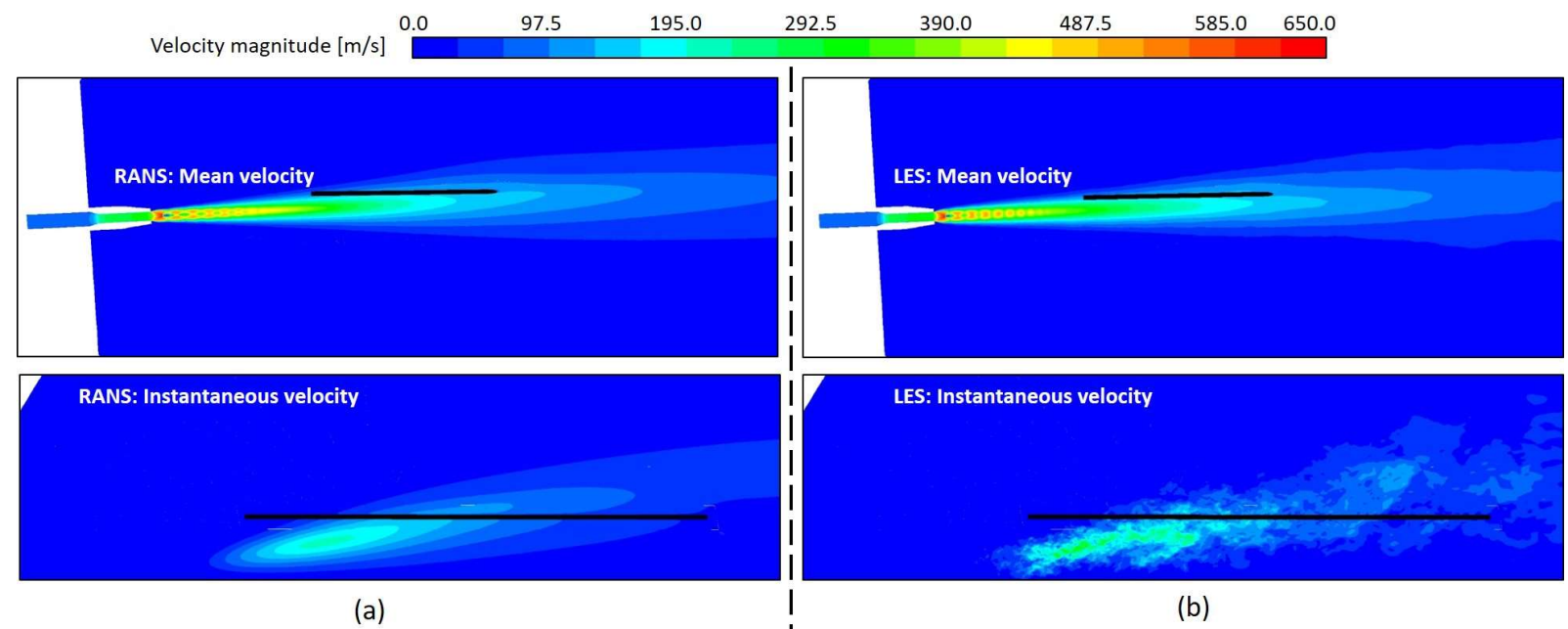

Figure 14. Comparison between the velocity field obtained from URANS (a) and LES (b).

From Figure 14 it can be observed that while the mean velocity for URANS and LES are quite similar, the instantaneous velocity field differs substantially. Furthermore, in none of the figures the presence of the interface is perceivable in the flow field, implying that the error introduced by the interpolation at the interface is very limited. In the bottom 2 images of Figure 14 it can also be noticed that the inserted cylinder covers the region where the largest forces will occur (high velocity region). 
Before discussing the calculated forces on the yarn it should be emphasized that in the simulations the yarn is considered to be at standstill. In reality the yarn is moving with a high axial velocity (x-direction), implying that the relative axial velocity of the air with respect to the yarn will be substantially lower in reality than considered here in the simulation. Consequently, the reported axial forces are maximal and it should be kept in mind that these will be lower in reality. Additionally, the transversal motion of the yarn will also have an effect on the forces it experiences, but the transversal velocity is not as high as the axial velocity. To obtain a spatial distribution of the forces on the yarn, the yarn was subdivided into rings. The resulting exterior force on each ring was calculated and stored every 5 time steps. An example of the force obtained from LES on one such segment is displayed in Figure 15 for the final 10000 time steps of the simulation. The segment was chosen at an orthogonal distance of 47D to the nozzle exit (the same distance as used in Figure 13). For each segment a time-averaged force and an RMSD value can be calculated. In Figure 16 the time-averaged force is displayed for both the LES and the URANS simulation. For the LES the forces were averaged over a period of 110000 time steps. For the URANS simulation the averaging was performed over a period of (only) 12000 time steps as the fluctuations in the forces are very small. Finally, Figure 17 visualizes the RMSD values for each component of the total force (sum of the shear and pressure force) along the yarn.

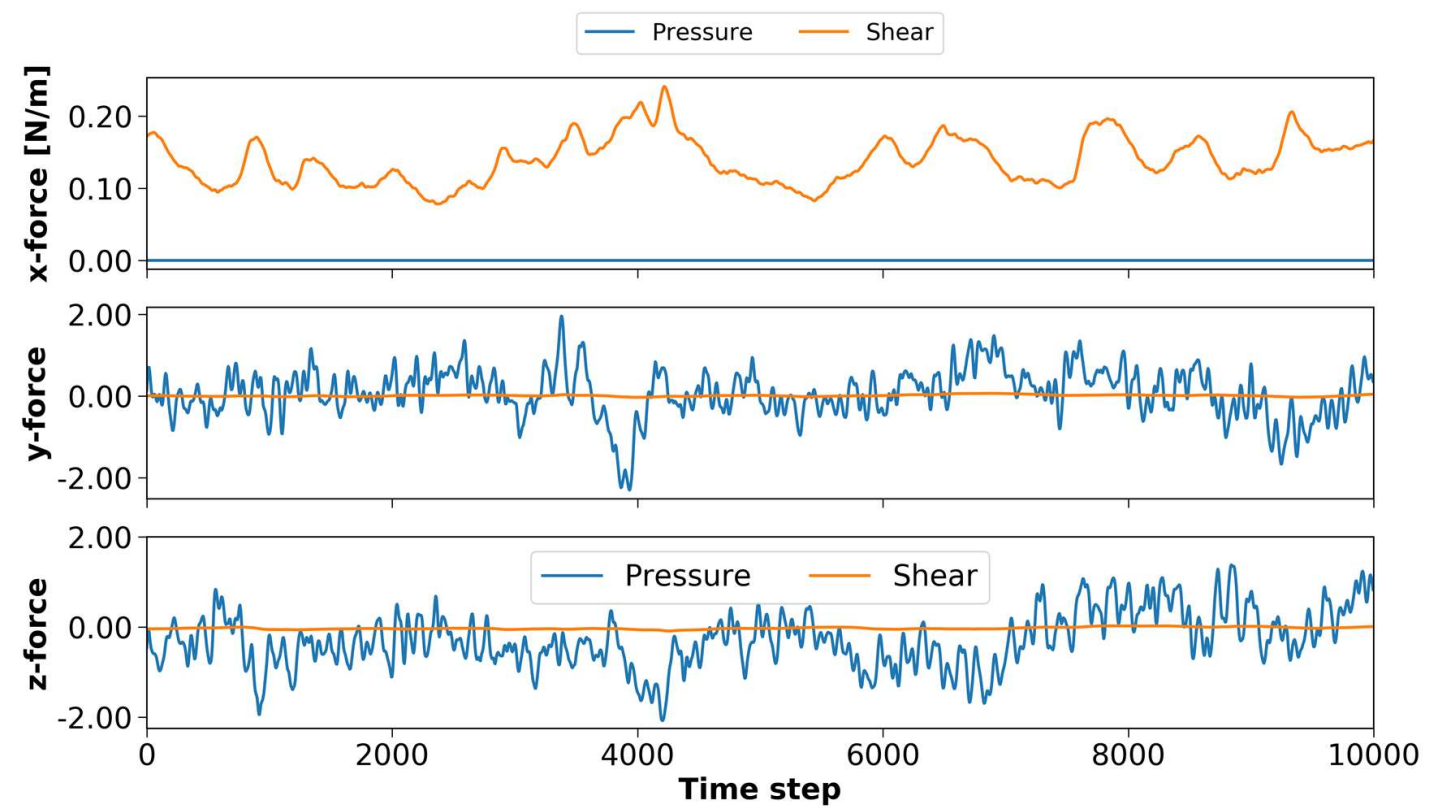

Figure 15. Force components as a function of time on a ring segment in the final 10000 time steps of the LES. 


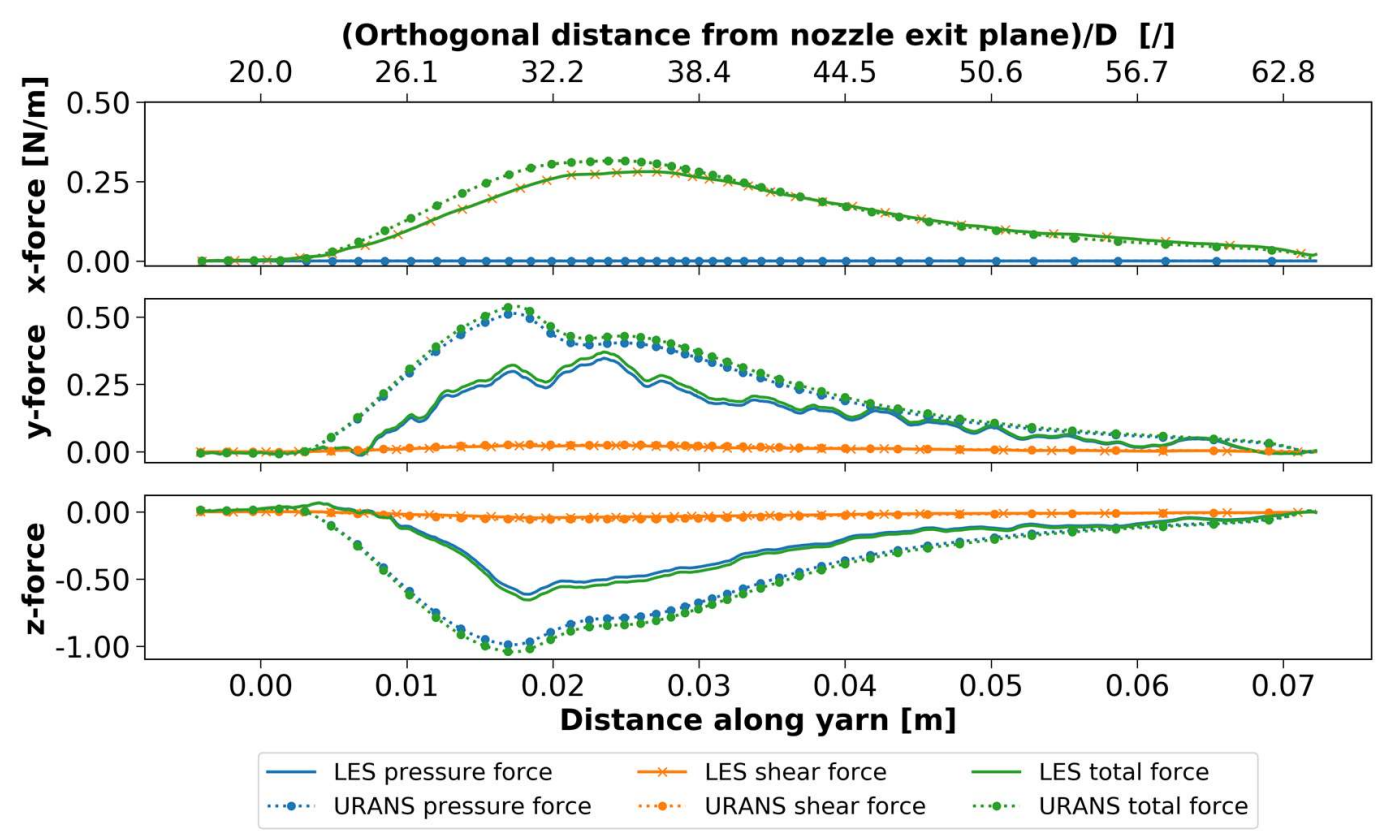

Figure 16. Time-averaged values for each component of the pressure and shear force.

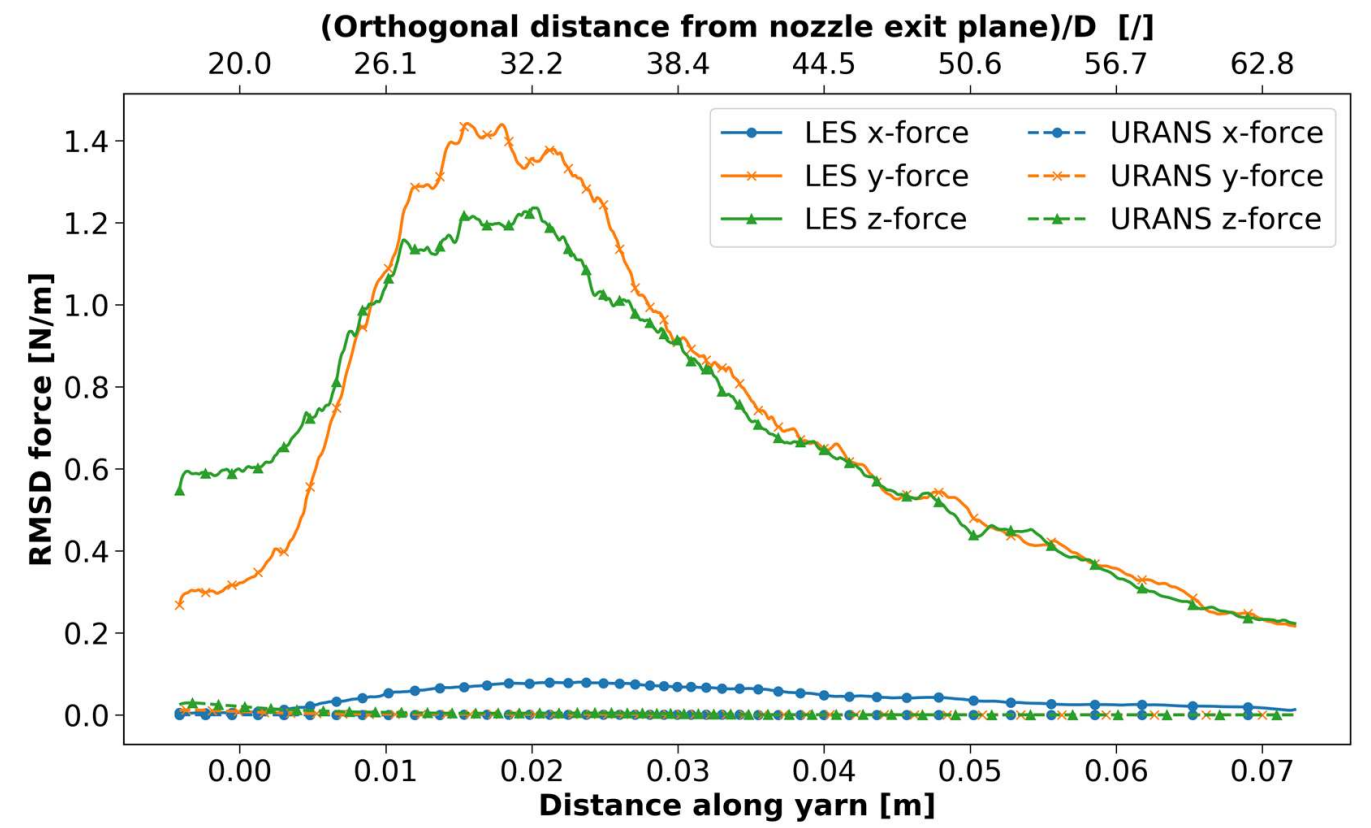

Figure 17. RMSD values for each component of the total force.

Figure 15 and Figure 16 clearly show that the force in the $\mathrm{x}$-direction is dominated by shear force, while the forces in the $\mathrm{y}$ - and $\mathrm{z}$-direction are dominated by pressure force. The average force in the $\mathrm{x}$-direction obtained from LES and URANS is very similar. The forces in the $y$ - and z-direction obtained from the LES still show some spatial oscillation. This is largely caused by the fact that the oscillations in the y- and zforces (Figure 17) are relatively large compared to the mean value (Figure 16). To remove this oscillation, the averaging should be performed over an even larger time period. Nevertheless, the overall trend is quite apparent: the magnitude of the average force obtained from LES is smaller than that obtained from URANS. 
To draw any sound conclusions about which of both simulations currently yields the most accurate average force would require a detailed experimental investigation. However, that is challenging due to the small forces.

From Figure 17 it can be observed that the URANS simulation yields an almost steady force. The maximal RMSD value was observed for the z-component and amounted to $0.032 \mathrm{~N} / \mathrm{m}$, which originates from a nonturbulence induced transient phenomenon. However, the LES illustrates that turbulence induced oscillations can far exceed this value especially in the orthogonal directions ( $\mathrm{y}$ and $\mathrm{z}$ ).

By comparing Figures 16 and 17, it can also be seen that the largest oscillations occur in the region with the largest mean force (high velocity region). Furthermore, RMSD values for the orthogonal forces are much larger than the mean value for that component. This implies that those force components switch sign from time to time. As can be seen in Figure 15, this is not unlikely to happen. The yarn tip does not experience a lot of tension and is, therefore, relatively free to move. Consequently, it is likely to be strongly affected by these force oscillations and might therefore behave very irregularly.

In Figure 17 it can be noticed that the transversal force oscillation reaches up to $1.4 \mathrm{~N} / \mathrm{m}$. This can be compared to oscillation amplitudes predicted by means of force coefficients and the velocity field obtained from the free jet simulation. To determine the transversal force coefficient we consider a cylinder subjected to cross-flow, for which a lot of experimental data is available. The maximal force oscillations were observed around $0.02 \mathrm{~m}$ downstream along the yarn. The Reynolds number at this location, based on the mean transversal velocity and the yarn diameter is about 1200 . This corresponds to a drag coefficient for a smooth cylinder of approximately 1 (see for example Welty et al. ${ }^{40}$ ). From the drag coefficient the transversal force coefficient (as employed by Osman et al. ${ }^{18}$ ) can be derived by multiplying it with the yarn diameter. This results in a transversal force coefficient of 7.2e-04 m. Multiplying this force coefficient by the dynamic pressure based on a density $\left(\sim 1.2 \mathrm{~kg} / \mathrm{m}^{3}\right)$ and the RMSD value of the transversal velocity $(31.7$ $\mathrm{m} / \mathrm{s}$ based on Figure 12) yields an estimate for the amplitude of the transversal force oscillations of 0.443 $\mathrm{N} / \mathrm{m}$ for a smooth yarn. This value is much smaller than the value of $1.4 \mathrm{~N} / \mathrm{m}$ obtained from the LES with the smooth yarn included in the flow domain. This indicates that the use of force coefficients in combination with the RMSD value of the velocity fluctuations yields in this case a significant underestimation of the fluctuations in the orthogonal forces due to turbulence.

In Figure 16 the mean axial force is at most $0.29 \mathrm{~N} / \mathrm{m}$. Compared to this, the value of $1.4 \mathrm{~N} / \mathrm{m}$ for the RMSD of the orthogonal force (or even the value $0.443 \mathrm{~N} / \mathrm{m}$ obtained with force coefficients) is quite substantial. Furthermore, in an air jet loom the weft is travelling at a certain velocity through the machine. The transversal forces, which are pressure-dominated, will nearly be unaffected by this motion. On the other hand, the axial force, which is dominated by shear, will be reduced. This was also verified by selecting a time step from the simulation, assigning a wall velocity to the cylinder and recalculating that time step. The reduction of the axial force due to the weft motion will reinforce the relative influence of the turbulent fluctuations on the transversal motion of the weft.

\section{Conclusion}

The current research has investigated the oscillations in the flow field of a relay nozzle jet and the corresponding force fluctuations experienced by a yarn based on large eddy simulations (LES). To the authors' best knowledge a similar investigation has not yet been performed in the context of air jet weaving.

The high supply pressure of the relay nozzles presents some challenges to the simulations due to compressibility effects. Compared to large eddy simulations on (highly) underexpanded jets found in literature the current model had to extend further into the far-field to capture the interaction with the weft, 
implying a substantial computational cost ( 43.5 days on 840 cores). The LES framework was successfully validated by comparing the centerline velocity and radial profiles of a free jet to data from experiments and literature. Additionally, several important aspects concerning the RANS simulation of highly underexpanded jets were highlighted.

The LES of a relay nozzle jet without the presence of a yarn clearly illustrated the oscillatory behavior of the flow. By using force coefficients, an initial estimate of the oscillations in the force experienced by the yarn could be made. As mentioned previously, it should be kept in mind that the reported forces are for a yarn at standstill. In reality, the yarn is moving and its motion will affect the forces it experiences. This is especially true for the axial forces as in reality the yarn travels with a high axial velocity, reducing the relative velocity of the air with respect to the yarn and thereby the forces. It was also shown that the relative influence of the turbulent fluctuations on the weft would be minimal if the weft were aligned with the jet axis.

In a second LES a rigid cylinder, representing the yarn, was inserted into the domain and the forces exerted on it were calculated. It was shown that the lengthwise force on the yarn was dominated by shear while the lateral forces were dominated by pressure. Furthermore, the use of force coefficients for prediction of turbulent oscillations in the force was found to be inaccurate. This is important when considering simulations aimed at calculating the motion of the weft and the stresses it experiences. Overall, the research has shown that the force oscillations cannot be neglected without further numerical and experimental research, to which this study can serve as a data source and/or a starting point.

Although admittedly the study in this paper still deviates a lot from the situation in a real air jet loom, the general conclusions concerning the turbulence in the jet still hold. The established model can serve as basis or guide towards future simulations with a closer resemblance to the real-life simulation and to analyze the effects of alterations, eventually leading to a better understanding of the weft behavior during insertion. In that light, some suggestions for future work can be found below.

\section{Future work}

Throughout the paper several differences between the simulated setup and the configuration in a real air jet loom have been mentioned. A continuation of this work could include adaptations to more closely represent the situation in a real machine.

A first point of attention was the use of a test nozzle instead of a real relay nozzle geometry. The curvature of a real relay nozzle close to the nozzle exit makes the flow asymmetric. This asymmetry will affect the quantitative values at the weft location. The influence of this simplification could be investigated by performing the simulation for a real nozzle geometry. However, the results from this simulation will be harder to validate. When changing the geometry to include the curvature, the same methodology as presented in this paper can be used, but one should pay attention to the alignment of the refinement cone and the mesh, which might depend on the supply pressure.

A second point of attention is the omission of the reed. This will undoubtedly affect the forces experienced by the weft. Turbulent vortices will still form due to the shear of the jet flow with the surrounding air and travel towards the reed channel and the yarn. Including the reed in the simulations and studying the interaction of the jet (turbulence) with the lamellae would be very interesting, but would require an enormous amount of computational resources. Therefore, a first step could be to include a simplified representation of the reed in the simulations. 
Another point of attention is that the inserted weft is currently a stationary, straight cylinder. To study the motion and the stresses of the weft, it would be desirable to perform FSI simulations with a flexible weft, which are unfortunately very resource intensive. One possibility would be to first perform a CFD simulation until quasi-steady state is reached and then switching to an FSI simulation for a limited amount of time steps to see the influence. Another possibility is to use the forces obtained from an LES and impose these to a structural model to observe the resulting motion of the weft and the stresses it experiences. Even though this neglects the change in position and orientation of the weft within the flow field and the interaction between the weft motion and the flow, it can still yield valuable information.

\section{References}

1. Adanur S. Air jet weaving. In: Handbook of weaving. Boca Raton, FL: CRC Press, 2001, pp.175-224.

2. Wu Z, Chen S, Liu Y, et al. Air-flow characteristics and yarn whipping during start-up stage of air-jet weft insertion. Text Res J 2016; 86: 1988-1999.

3. Vangheluwe L. Air-jet weft insertion. Text Prog 1999; 29: 1-63. DOI: 10.1080/00405169908688878.

4. Uno M. A study on air-jet loom with substreams added, part 1: Deriving the equation of motion for weft. J Textile Mach Soc Jpn 1972; 25: 48-56.

5. Nosraty $\mathrm{H}$, Jedi AAA and Mousaloo Y. Simulation analysis of weft yarn motion in single nozzle air-jet loom to study the effective parameters. Indian J Fibre Text Res 2008; 33: 45-51.

6. Celik N, Babaarslan $\mathrm{O}$ and Bandara MPU. A mathematical model for numerical simulation of weft insertion on air-jet weaving machine. Text Res J 2004; 74: 236-240. DOI: 10.1177/004051750407400309.

7. Oh TH, Kim SD and Song DJ. A numerical analysis of transonic/supersonic flows in the axisymmetric main nozzle of air-jet loom. Text Res J 2001; 71: 783-790.

8. Kim HD, Lim C-M, Lee H-J, et al. A study of the gas flow through air jet loom. J Therm Sci 2007; 16: 159-163.

9. Belforte $\mathrm{G}$, Mattiazzo G, Viktorov V, et al. Numerical model of an air-jet loom main nozzle for drag forces evaluation. Text Res J 2009; 79: 1664 - 1669.

10. Jin Y, Cui J, Zhu L, et al. An investigation of some parameter effects on the internal flow characteristics in the main nozzle. Text Res J 2016; 87: 91-101.

11. He $\mathrm{S}$, Qian $\mathrm{Y}$, Xue $\mathrm{W}$, et al. Numerical simulation of flow field in air-jet loom main nozzle. AUTEX Research Journal 2018; 19: 1-10.

12. Kim JH, Setoguchi T and Kim HD. Numerical study of sub-nozzle flows for the weft transmission in an air jet loom. In: 6th BSME International conference on Thermal engineering Dhaka, Bangladesh, 2015, pp.264-269. Elsevier.

13. Grassi C, Schöter A, Gloy Y-S, et al. Increasing the energy efficiency of air jet weaving based on a novel method to exploit energy savings potentials in production processes of the textile industry. Journal of Environmental Science and Engineering 2016; B5: 26-34. 
14. Münkel A, Gloy YS and Gries T. Development and testing of a relay nozzle concept for air-jet weaving. IOP Conference series: Materials Science and Engineering. Corfu, Greece: IOP Publishing, 2017, p. 1-6.

15. De Meulemeester S, Puissant P and Van Langenhove L. Three-dimensional simulation of the dynamic yarn behavior on air-jet looms. Text Res J 2009; 79: 1706-1714.

16. Delcour L, Peeters J and Degroote J. Three-dimensional fluid-structure interaction simulations of a yarn subjected to the main nozzle flow of an air-jet weaving loom using a Chimera technique. Text Res J 2019; 0: 1-19.

17. Osman A, Delcour L, Hertens I, et al. Toward three-dimensional modeling of the interaction between the air flow and a clamped-free yarn inside the main nozzle of an air jet loom. Text Res J 2018; 0: 112.

18. Osman A, Malengier B, De Meulemeester S, et al. Simulation of air flow-yarn interaction inside the main nozzle of an air jet loom. Text Res J 2017; 88: 1173-1183. DOI: 10.1177/0040517517697646.

19. Pei $Z$ and Yu C. Numerical study on the effect of nozzle pressure and yarn delivery speed on the fiber motion in the nozzle of Murata vortex spinning. J Fluids Struct 2011; 27: 121-133.

20. Zeng YC and Yu CW. Numerical simulation of fiber motion in the nozzle of an air-jet spinning machine. Text Res J 2004; 74: 117-122.

21. Franquet $\mathrm{E}$, Perrier $\mathrm{V}$, Gibout $\mathrm{S}$, et al. Free underexpanded jets in a quiescent medium: A review. Prog Aerosp Sci 2015; 77: 25-53.

22. Lin $\mathrm{P}, \mathrm{Xu} \mathrm{W}$, Jin $\mathrm{Y}$, et al. Simulation of motion of long flexible fibers with different linear densities in jet flow. Journal of Nanotechnology 2018; 2018: 1-10.

23. Oosthuizen PH and Carscallen WE. Introduction to compressible fluid flow. CRC Press (Taylor \& Francis Group), 2013.

24. Donaldson CD and Snedeker RS. A study of free jet impingement. Part 1. Mean properties of free and impinging jets. J Fluid Mech 1971; 45: 281-319.

25. Adamek K, Karel P, Kolar J, et al. Relay nozzles and weaving reed. International Journal of Mechanical Engineering and Applications 2015; 3: 13-21.

26. Osman A. Computational fluid-structure interaction study of the aeroelastic behavior of a wire in transonic and supersonic flows. PhD thesis, Ghent University, 2017.

27. Wilcox DC. Dilatation-dissipation corrections for advanced turbulence models. AIAA 1992; 30: 26392646.

28. Zeman O. Dilatation dissipation: The concept and application in modeling compressible mixing layers. Phys. Fluids 1990; 2: 178-188. 
29. Sarkar S, Erlebacher G, Hussaini MY, et al. The analysis and modelling of dilatational terms in compressible turbulence. J Fluid Mech 1991; 227: 473-493.

30. Yoder DA, DeBonis JR and Georgiadis NJ. Modeling of turbulent free shear flows. Comput Fluids 2015; 117: 212-232.

31. Yüceil KB and Ötügen MV. Scaling parameters for underexpanded supersonic jets. Phys. Fluids 2002; 14: 4207-4215.

32. Kaushik M, Kumar R and Humrutha G. Review of computational fluid dynamics studies on jets. American Journal of Fluid Dynamics 2015; 5: 1-11.

33. Celik IB, Cehreli ZN and Yavuz I. Index of resolution quality for large eddy simulations. J Fluids Eng 2005; 127: 949-958.

34. Vuorinen V, Yu J, Tirunagari S, et al. Large-eddy simulation of highly underexpanded transient gas jets. Phys. Fluids 2013: 1-21.

35. Hamzehloo A and Aleiferis PG. Large eddy simulation of highly turbulent under-expanded hydrogen and methane jets for gaseous-fuelled internal combustion engines. Int J Hydrogen Energy 2014; 39: 21275-21296.

36. Li X, Zhou R, Yao W, et al. Flow characteristic of highly underexpanded jets from various nozzle geometries. Appl Therm Eng 2017; 125: 240-253. DOI: 10.1016/j.applthermaleng.2017.07.002.

37. Gong L, Duan Q, Liu J, et al. Spontaneous ignition of high-pressure hydrogen during its sudden release into hydrogen/air mixtures. Int J Hydrogen Energy 2018; 43: 23558-23567.

38. Li X, Yao W and Fan X. Large-eddy simulation of time evolution and instability of highly underexpanded jets AIAA 2016; 54: 3191-3211.

39. Goldstein RJ. Inviscid, incompressible fluids. In: Brenda Brienza KZaEM (ed) Fluid mechanics measurements. USA: Hemisphere Publishing Corporation, 1983, pp.51 - 55.

40. Welty JR, Wicks CE, Wilson RE, et al. Chapter 12: Viscous flow. In: Fundamentals of momentum, heat and mass transfer. 5 ed. USA: John Wiley \& Sons, Inc., 2008.

41. Pope SB. Turbulent Flows. New York, USA: Cambridge University Press, 2000. 


\section{Appendix}

\section{The (dynamic) Smagorinsky model.}

In order to better frame the dynamic Smagorinsky model, a paragraph on RANS modelling is included first. The explanation is largely based on the work of Pope. ${ }^{\mathrm{A}}$

The RANS equations are obtained by applying a statistical averaging to the Navier-Stokes equations. The velocity field is decomposed into a mean-value $\left(U_{i}\right)$ and a random, fluctuating component $\left(u_{i}\right)$. This fluctuating component corresponds to turbulent fluctuations of the flow. In the equations obtained after performing the statistical averaging, some terms with $\left\langle u_{i} u_{j}\right\rangle$ remain (with \langle\rangle indicating the mean value). These terms pose a closure problem which is resolved by modelling these terms. Thus, in RANS models all chaotic turbulent fluctuations are modelled and not resolved. However, this does not imply that RANS models are steady-state models; transient terms can still be present in the equations. Transient RANS models are often referred to as unsteady RANS or URANS models. In URANS models the chaotic turbulent fluctuations are still not resolved, but intrinsic transient phenomena such as vortex shedding and timevarying boundary conditions can be calculated.

The (dynamic) Smagorinsky model is an LES model. In LES models a spatial-filtering is applied to the Navier-Stokes equations. The velocity field is then decomposed as the sum of a filtered value $\left(\bar{U}_{i}\right)$ and a subgrid-scale component. For an incompressible fluid the filtered momentum equation becomes:

$\frac{\delta \bar{U}_{i}}{\delta t}+\frac{\delta \overline{U_{i} U_{j}}}{\delta x_{i}}=v \frac{\delta^{2} \bar{U}_{j}}{\delta x_{i} \delta x_{i}}-\frac{1}{\rho} \frac{\delta \bar{p}}{\delta x_{j}}$

and the residual stress tensor, which makes these equations different from the Navier-Stokes equations, is defined as:

$\tau_{i j}^{R} \equiv \overline{U_{i} U_{j}}-\bar{U}_{i} \bar{U}_{j}$

The anisotropic component $\left(\tau_{i j}^{r}\right)$ of this residual-stress tensor poses a closure problem and needs modelling (the isotropic component is included in a modified pressure term). As stated by Pope ${ }^{\mathrm{A}}$ : the main difference between the LES equations and the RANS equations is that in LES the fields $\bar{U}_{i}, \bar{p}$ and $\tau_{i j}^{r}$ are chaotic, 3dimensional unsteady fields even when the flow is statistically stationary or homogeneous. Thus, in LES turbulent fluctuations larger than a scale determined by the chosen filter width are resolved and only the ones smaller than the filter width are modelled.

In the Smagorinsky model the aforementioned anisotropic component is modelled as follows:

$$
\begin{aligned}
& \bar{S}_{i j} \equiv \frac{1}{2}\left(\frac{\delta \bar{U}_{i}}{\delta x_{j}}+\frac{\delta \bar{U}_{j}}{\delta x_{i}}\right) \\
& \bar{S} \equiv\left(2 \bar{S}_{i j} \bar{S}_{i j}\right)^{1 / 2} \\
& \tau_{i j}^{r}=-2\left(C_{s} \Delta\right)^{2} \bar{S} \cdot \bar{S}_{\mathrm{ij}}
\end{aligned}
$$

In equation (A5) $C_{S}$ is a constant (the Smagorsinky constant) and $\Delta$ is the selected filter width, often based on the local mesh size. Unfortunately, the value that should be used for $C_{s}$ differs depending on the flow regime. ${ }^{\mathrm{A}}$ In the dynamic Smagorinsky model the coefficient $\left(C_{S}\right)$ is not constant; instead it is computed based on information from the resolved scales of motion. Without going into detail, a second filter (the test

\footnotetext{
A Pope SB. Turbulent Flows. New York, USA: Cambridge University Press, 2000.
} 
filter) is considered, typically with twice the width of the original filter. The coefficient $C_{s}$ is then calculated based on the difference between the flow field obtained with the original filter and the flow field obtained with the test filter.

\section{LES quality criteria}

\section{Ratio of turbulent kinetic energy}

The quality of an LES is often evaluated by estimating the fraction of turbulent kinetic energy that is modelled (and thus not resolved) by the simulation. As proposed by Pope ${ }^{\mathrm{B}}$ one should aim at resolving more than $80 \%$ of the total turbulent kinetic energy. The resolved turbulent kinetic energy $\left(k_{\text {res }}\right)$ is calculated from the root-mean-square of the velocity fluctuations according to Formula (A6). The subgridscale turbulent kinetic energy $\left(k_{s g s}\right)$ is estimated from the calculated subgrid-scale viscosity $\left(\mu_{s g s}\right)$ using Formula (A7). In this formula $C_{D S}$ is the local value of the dynamic Smagorinsky constant and $V_{\text {cell }}$ is the local cell volume. The ratio of turbulent kinetic energy $\left(k_{\text {ratio }}\right)$ is then obtained from Formula (A8) and preferably exceeds 0.8 .

$$
\begin{aligned}
& k_{\text {res }}=\frac{1}{2}\left(v_{x, r m s}^{2}+v_{y, r m s}^{2}+v_{z, r m s}^{2}\right) \\
& k_{s g s}=\left(\frac{1}{C_{D S}} \cdot \frac{\mu_{s g s}}{\rho V_{\text {Cell }}^{1 / 3}}\right)^{2} \\
& k_{\text {ratio }}=\frac{k_{\text {res }}}{k_{\text {res }}+k_{\text {sgs }}}
\end{aligned}
$$

A contour plot of the mean turbulent kinetic energy ratio for the relay nozzle jet is shown in Figure A1. After allowing the LES to reach a semi-steady state the ratio was calculated and its value averaged over 40500 time steps. The cone encompassing the fine mesh is also drawn on the figure, white regions indicate zones where the ratio is below 0.7 . From the figure it can be seen that the ratio satisfies the criterion within the cone and somewhat beyond its borders.

\footnotetext{
B Pope SB. Large-eddy simulation. In: Turbulent Flows. New York: Cambridge University Press, 2000, pp. 558641.
} 


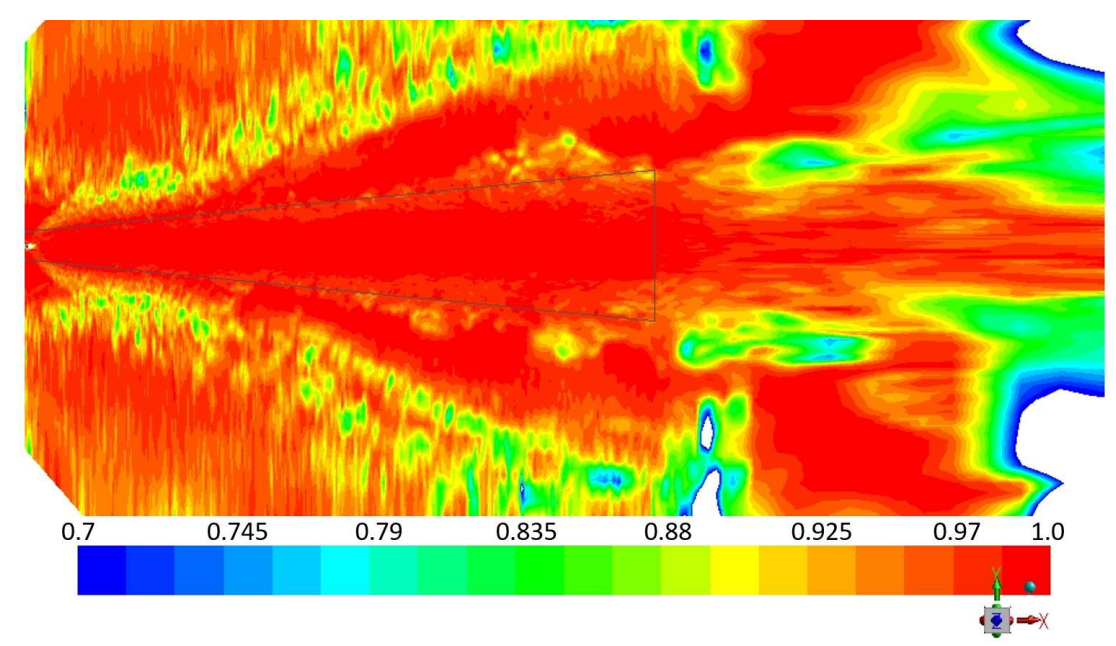

Figure A1: Contour plot of the mean turbulent kinetic energy ratio. In the white regions the ratio is smaller than 0.7 .

\section{Relative effective viscosity index}

The relative effective viscosity index, also referred to as index of resolution quality or $L E S_{-} I Q_{v}$, was introduced by Celik et al. ${ }^{\mathrm{C}}$. The criterion evaluates the relative contribution of the subgrid and numerical viscosity. The numerical viscosity is, however, unknown and an estimate of the numerical error would require simulations on multiple grids. Nevertheless, the criterion can still give an indication of whether or not the used grid is sufficiently fine by omitting the numerical viscosity. The index is calculated according to Formula (A9) based on Celik et al. ${ }^{\mathrm{D}}$ with $v$ the laminar viscosity and $v_{s g s}$ the subgrid scale viscosity. A value higher than 0.8 is considered a good LES and a value exceeding 0.95 corresponds, essentially, to direct numerical simulation (DNS). A contour plot of the mean value is displayed in Figure A2, the mean value is obtained similarly to the ratio of turbulent kinetic energy. Based on this criterion the current LES has a good resolution even in regions beyond the cone.

$$
L E S_{-} I Q_{v}=\frac{1}{1+0.05\left[\frac{v+v_{s g s}}{v}\right]^{0.53}}
$$

\footnotetext{
C Celik IB, Cehreli ZN and Yavuz I. Index of Resolution Quality for Large Eddy Simulations. Journal of Fluids Engineering. 2005; 127: 949-58.

${ }^{D}$ Celik IB, Klein M, Freitag M and Janicka J. Assessment measures for URANS/DES/LES: an overview with applications. Journal of Turbulence. 2006; 7: 1-7.
} 


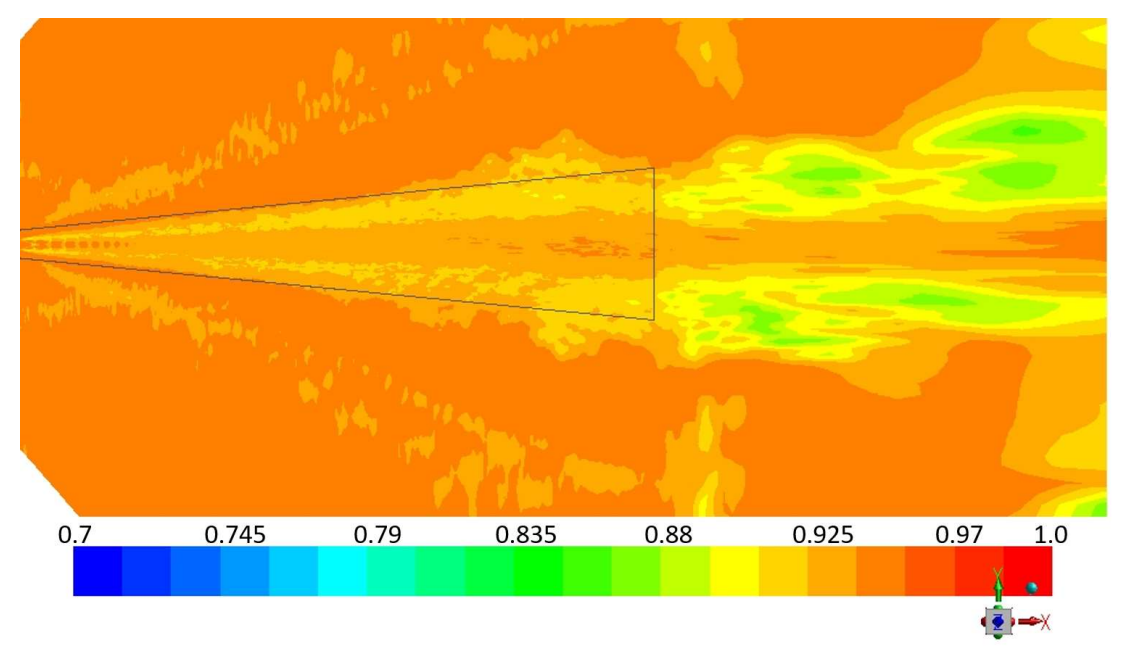

Figure A2: Contour plot of the mean turbulent kinetic energy ratio. In the white regions the ratio is smaller than 0.7 .

\section{Turbulent energy spectra}

Another commonly used quality criterion for LES is based on the turbulent energy spectrum. Following e.g. Pope ${ }^{\mathrm{E}}$, the turbulent energy spectrum can be split up in roughly 3 categories in terms of eddy size. The largest eddies contain the most energy and form the "energy-containing range". Energy from these eddies is transferred to very small eddies, where it is dissipated by viscous forces. These small eddies constitute the "dissipation range". In between is the "inertial subrange" which is determined by inertia effects and transfers energy from the large scales to successively smaller scales. Using Kolmogorov's hypothesis it can then be shown that the inertial subrange exhibits, on a log-log-scale, a $-5 / 3$ decay rate.

The quality of the LES is then evaluated by verifying that the turbulent energy spectrum retrieves at least part of the inertial subrange by looking for the $-5 / 3$ decay rate. Theoretically, the energy spectra should be calculated using spatial correlations. However, if Taylor's frozen turbulence hypothesis is assumed to apply, the energy spectrum can be calculated based on the velocity fluctuations at a fixed location in space. In this research, the velocity data was exported at every time step in 15 points. These points are visualized as white dots in Figure A3, the axial distances are 20D, 30D, 45D, 60D and 75D. The energy spectra for the points closest to the nozzle exit and furthest from the nozzle exit are show in Figure A4 and Figure A5, respectively. With reference to Figure $A 3$, the label $Y_{0}$ is located on the nozzle axis, $Y_{1}$ is at an intermediate radial distance and $\mathrm{Y}_{2}$ corresponds to the point at the largest radial distance. The spectra were obtained using the final 24000 time steps from the simulation. Apart from the point with the largest axial and radial distance from the nozzle exit, the $-5 / 3$ decay rate can be observed, satisfying the quality criteria.

\footnotetext{
${ }^{\text {E }}$ Pope SB. Turbulent Flows. New York: Cambridge University Press, 2000.
} 


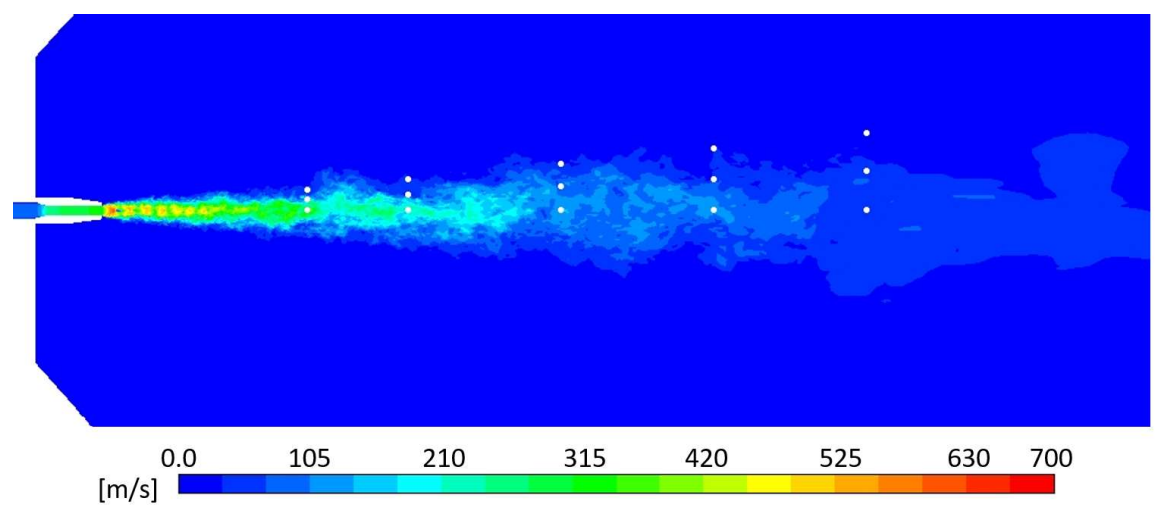

Figure A3: White dots indicate the points used for the turbulent energy spectra and two-point correlations displayed on top of a contour plot of the instantaneous velocity magnitude.

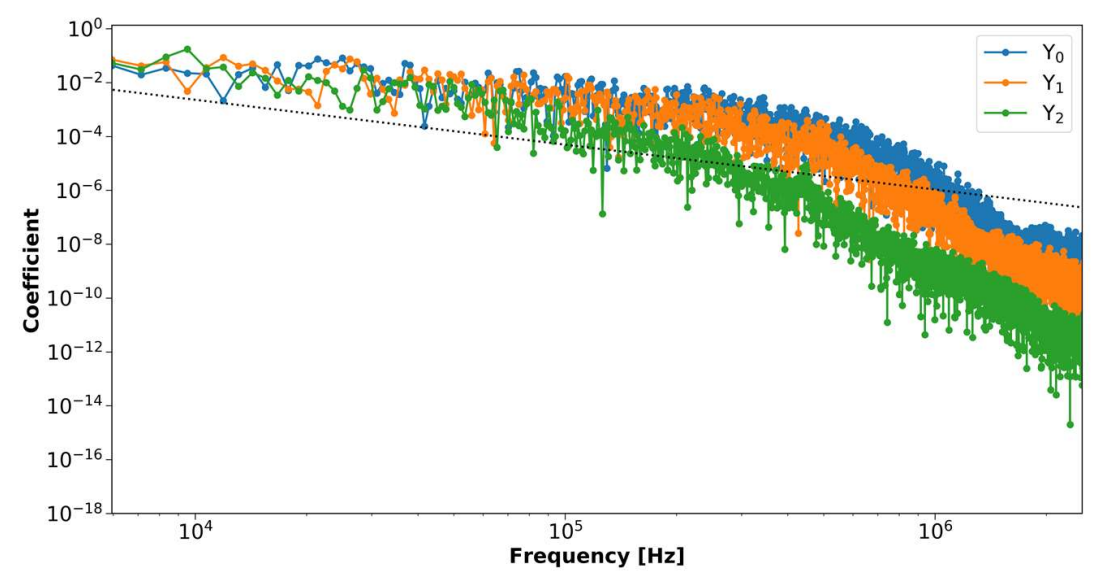

Figure A4: Energy spectra for the points at an axial distance of 20D from the nozzle exit. The radial distance increases from $Y_{0}$ to $Y_{1}$ to $Y_{2}$. The dashed line indicates the $-5 / 3$ slope.

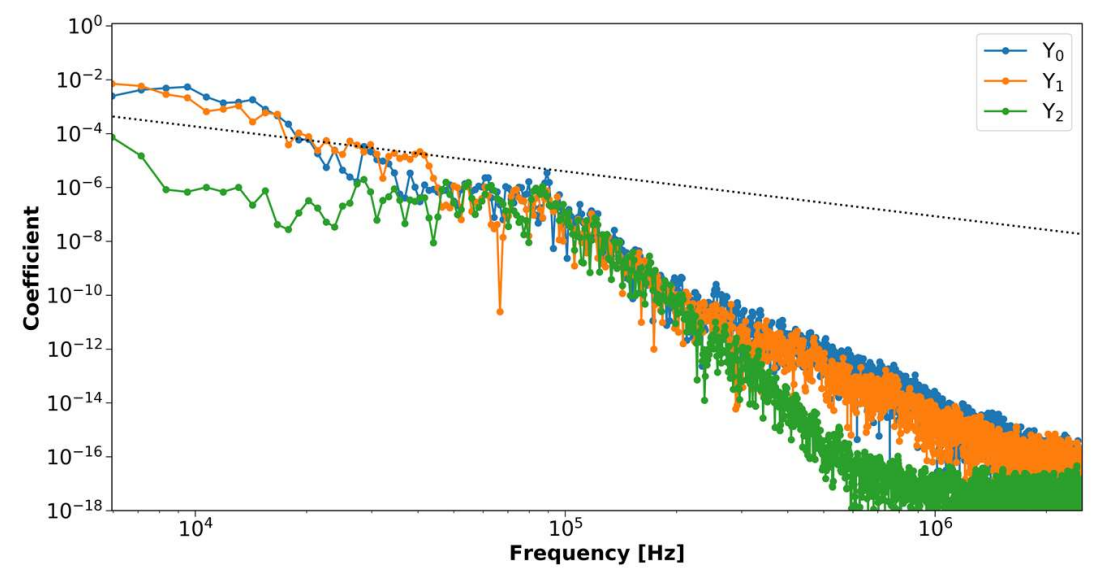

Figure A5: Energy spectra for the points at an axial distance of 75D from the nozzle exit. The radial distance increases from $\mathrm{Y}_{0}$ to $\mathrm{Y}_{1}$ to $\mathrm{Y}_{2}$. The dashed line indicates the $-5 / 3$ slope.

\section{Two-point correlations}

The final quality criterion evaluated for the LES is based on the velocity correlation between a point and the points in its vicinity. The correlation provides information about the cell size relative to the size of the 
largest eddies. To properly resolve the large eddies it is desired that the two-point correlation normalized by the square of rms-value of the velocity fluctuations exceeds 0.1 for at least 8 cells (see for example Davidson $^{\mathrm{F}}$ ). The correlations are calculated both in the axial and the radial direction for the same points as used for the energy spectra (See Figure A3). The axial velocity correlation (correlation between axial velocity fluctuations in the axial direction) for all points is plotted in Figure A6 and the radial correlation (correlation between radial velocity fluctuations in the radial direction) in Figure A7. The vertical red lines indicate the 8-cell limit. The figures show that the criterion is satisfied both in the radial and axial direction apart from the axial correlation in the points at a distance of 75D, which is outside the region of interest.

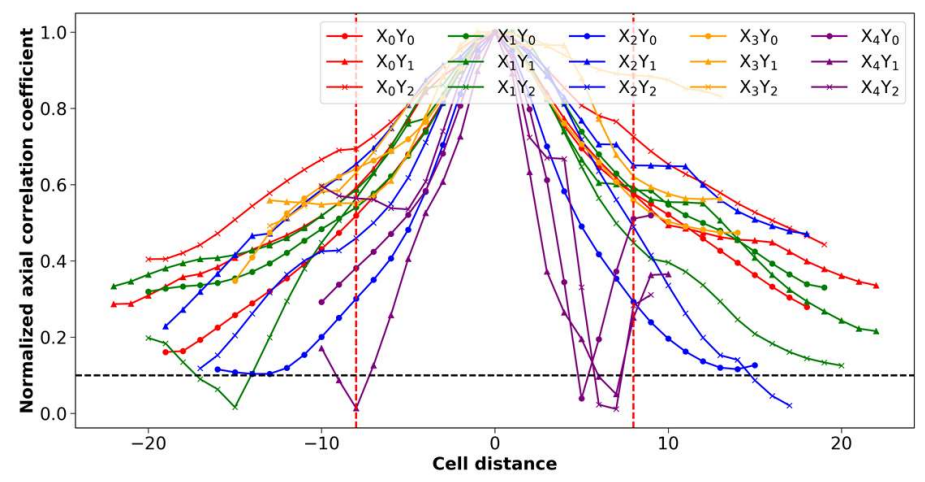

Figure A6: Axial velocity correlation. The points correspond to those in Figure A3. The axial distance increases from $\mathrm{X}_{0}$ to $\mathrm{X}_{4}$. For a selected axial distance the radial distance increases for $\mathrm{Y}_{0}$ to $\mathrm{Y}_{2}$.

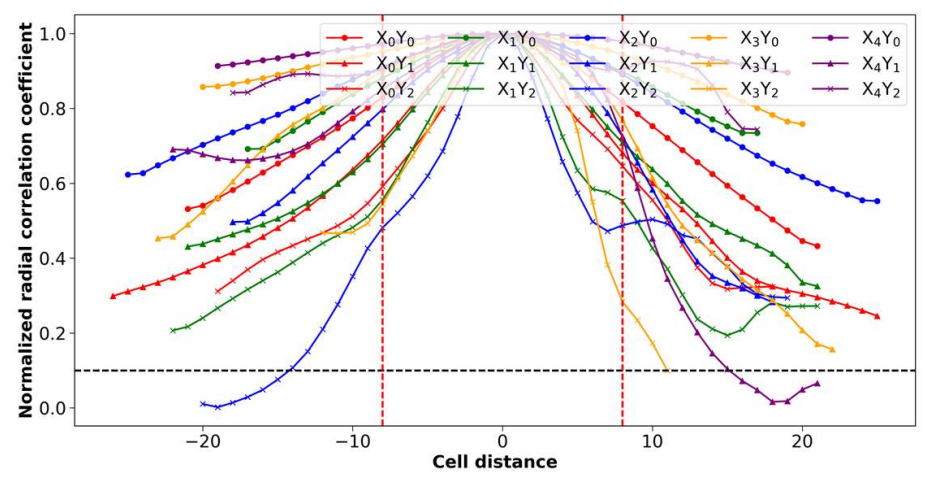

Figure A7: Radial velocity correlation. The points correspond to those in Figure A3. The axial distance increases from $\mathrm{X}_{0}$ to $\mathrm{X}_{4}$. For a selected axial distance the radial distance increases for $\mathrm{Y}_{0}$ to $\mathrm{Y}_{2}$.

\section{Conclusion about the quality criteria}

From the results shown above it can be concluded that within the refinement region (up to 70D) the grid is of sufficiently high quality but that the quality quickly decays outside of the refinement cone.

\footnotetext{
F Davidson L., Large eddy simulations: How to evaluate resolution. International Journal of Heat and Fluid Flow. 2009; 30(5): 1016-25.
} 